\title{
Planning and Implementing Trajectories for Autonomous Underwater Vehicles to Track Evolving Ocean Processes based on Predictions from a Regional Ocean Model
}

\author{
Ryan N. Smith*i, Yi Chao \\ David A. Caron§ Burton H. Jones ${ }^{\S}$ and Gaurav S. Sukhatme*
}

\begin{abstract}
Trajectory design for Autonomous Underwater Vehicles (AUVs) is of great importance to the oceanographic research community. Intelligent planning is required to maneuver a vehicle to high-valued locations for data collection. We consider the use of ocean model predictions to determine the locations to be visited by an AUV. While traversing the path connecting these locations, the vehicle collects data and provides near-real time, in situ measurements back to the model to increase the skill of future predictions. Our focus is on algorithms to determine relevant points of scientific interest for a vehicle to visit within a dynamically evolving oceanographic feature. This represents a first approach to an end to end autonomous prediction and tasking system for aquatic, mobile sensor networks. We design sampling missions and present simulated scenarios and results from field deployments for AUV feature tracking in the Southern California coastal ocean.
\end{abstract}

\section{Introduction}

More than three-fourths of our earth is covered by water, yet we have explored less than $5 \%$ of the aquatic environment. Autonomous Underwater Vehicles (AUVs) are a key tool in this exploration. To make new discoveries and improve our overall understanding of the ocean, scientists must make use of these platforms by implementing effective monitoring and sampling techniques. One example of intelligent ocean sampling is the coordinated control of Autonomous and Lagrangian Sensor Platforms (ALPS), developed in the series of articles: (Fiorelli, Leonard, Bhatta, Paley, Bachmayer \& Fratantoni 2006), (Leonard, Paley, Lekien, Sepulchre, Fratantoni \& Davis 2007), (Paley, Zhang \& Leonard 2007) and (Paley, Zhang \& Leonard 2008). Such research efforts have opened the door for the design and implementation of adaptive, mobile sensor platforms and networks to aid in the study of complex phenomena such as ocean currents, tidal mixing and other dynamic ocean processes.

To this end, three laboratories at the University of Southern California (USC) have formed CINAPS (pronounced [sin-aps]); the Center for Integrated Networked Aquatic PlatformS. The mission of this collaborative research group is to bridge the gap between technology, communication, and the scientific exploration of local and regional aquatic ecosystems through the implementation of an embedded sensor network along the Southern California coast (Smith, Das, Heidarsson, Pereira, Cetinić, Darjany, Ève Garneau, Howard, Oberg, Ragan, Schnetzer, Seubert, Smith, Stauffer, Toro-Farmer, Caron, Jones \& Sukhatme 2009c). This infrastructure is designed for facilitation of long-term, in-depth, multi-faceted investigation of physical, chemical and biological processes resulting from coastal urbanization and climate change. One component of this network, and the focus of this paper, are mobile sensor platforms in the form of Autonomous Slocum Gliders (ASGs) (Webb Research Corporation 2008). Based on their deployment longevity, and the use of multiple gliders (see e.g., (Davis, Ohman, Rudnick, Sherman \& Hodges 2008)), these vehicles can provide an extended spatio-temporal series of observations (details in

\footnotetext{
*Robotic Embedded Systems Laboratory, University of Southern California, Los Angeles, CA 90089 USA

${ }^{\dagger}$ Corresponding author, email: ryannsmi@usc.edu

${ }^{\ddagger}$ Jet Propulsion Laboratory, California Institute of Technology, 4800 Oak Grove Drive, Pasadena, CA 91109 USA

${ }^{\S}$ Department of Biological Sciences, University of Southern California, Los Angeles, CA 90089 USA
} 
Sect. 4). This study investigates a method to produce trajectories for a multi-vehicle application for ASGs in the Southern California coastal ocean.

The trajectory design method presented is based upon predictions from a regional ocean model. As complex and understudied as the ocean may be, we are still able to model and predict certain behaviors moderately well over short time periods. Consistently comparing model predictions with collected data, and adjusting for discrepancies, will increase the range of validity of existing ocean models, both temporally and spatially.

Utilizing the available technology and infrastructure, we consider the problem of integrating ocean model predictions into the path planning and trajectory design procedure for AUVs, with the goal of tracking and sampling within an interesting and evolving ocean feature. Collecting data for ocean science can be extremely hit-or-miss, both temporally and spatially, especially when one is interested in a specific biogeochemical event. In addition, areas of scientific interest within the ocean dynamically move and evolve. Thus, continuously operating a sensor platform (static or mobile) in a pre-designated and confined sampling area is not the most effective technique to gather data for analysis and assessment of ocean processes which may occur sporadically, and dynamically propagate throughout the ocean. Here we aim to increase the likelihood of gathering data of high scientific merit by deriving the sampling locations from a prediction of the evolution of a given feature of interest. We build upon the threedimensional (two spatial dimensions plus time) tracking algorithm presented in (Smith, Chao, Jones, Caron, Li \& Sukhatme 2009a), with the intention of generating a mission plan that accurately steers multiple ASGs to locations of high scientific interest within an evolving ocean feature. The primary contributions of this paper are the development of an innovative toolchain, and the waypoint generation algorithms for the practical application of AUV path planning and trajectory design.

The goal of this study is to present an innovative ocean sampling method that utilizes model predictions and ASGs to collect interesting oceanographic data that can also increase model skill. Our motivation is to track and collect daily information about an ocean process or feature which has a lifespan on the order of weeks. Based on the interesting biochemical ocean dynamics presented in Sect. 2, in addition to its proximity to our laboratories at USC, we choose to focus our research on an oceanographic region referred to as the Southern California Bight $(\mathrm{SCB})^{1}$. The SCB is denoted by the black box in Fig. 1(a), with an enlarged view in Fig. 1(b).

The mission plan to track and monitor dynamically evolving ocean processes or features is iteratively generated as follows. First, we identify a feature of interest in the SCB via direct observation or through remotely sensed data (e.g., satellite imagery). We then use a regional ocean model to predict the behavior of this feature, e.g., outfall from a waste water treatment plant, over a short time period, such as one day. This prediction is used to generate a sampling plan for deployed ASG(s) that steers the vehicle(s) to regions of scientific interest, based upon the given feature and its predicted evolution. Throughout execution of the sampling plan, the collected data are transmitted via the aforementioned network and assimilated into the ocean model. Incorporating the in situ ground truth, a new prediction is generated. This entire process is repeated until the feature dissipates or is no longer of interest.

We begin our discussion with a description of the primary research focus related to this study, a Harmful Algal Bloom (HAB). An algal bloom, and in particular a HAB, is a rapid increase of biomass of phytoplankton or cyanobacteria (potentially toxin producing species) caused by the addition of nutrients to and/or an alteration in the chemical properties of the ocean. Nutrients can be added to the coastal ocean via river runoff or waste water outfalls. Ocean chemistry can be altered by the addition of freshwater from these events, as well as by ocean processes such as an eddy or upwelling.

We continue our discussion with definitions of the sensor platform and ocean model considered, and a review of previous work related to similar problems. Section 5 contains an in-depth discussion and statement of the path planning problem, and describes the two main algorithms designed to obtain our solution; waypoint selection algorithm for both a boundary tracking and centroid tracking mission scenario. We design sampling plans and present simulated and implemented experimental results for AUV retasking in Southern California coastal waters. We conclude with an analysis of the experimental results and present areas of current and future investigation. A crucial component to this study is the validation of this toolchain via at-sea trials. Extensive deployment time $(>1500 \mathrm{~km}$ traversed during more than 100 days at sea from January 2009 - September 2009) has provided several successful validation results. Here we present two examples of feature-tracking missions implemented onto deployed AUVs.

${ }^{1}$ The SCB is the oceanic region contained within $32^{\circ} \mathrm{N}$ to $34.5^{\circ} \mathrm{N}$ and $-117^{\circ} \mathrm{E}$ to $-121^{\circ} \mathrm{E}$. 


\section{Background and Motivation}

\subsection{Southern California Ocean Dynamics}

The motivation for using predictive capabilities to design trajectories with the intent of tracking an evolving ocean feature is derived from a practical problem that exists in many coastal communities around the world, and in particular, Southern California. As the rate of urbanization in coastal communities continues to increase, land use and land cover (i.e., significant increase in impervious surfaces) in these areas are permanently altered. This alteration affects both the quantity of freshwater runoff, and its particulate and solute loadings, which has an unknown impact (physically, biogeochemically, biologically and ecologically) on the coastal ocean (Warrick \& Fong 2004). One documented result of these impacts is an increase in the occurrence of algal and phytoplankton blooms. Such biological phenomena are a primary research interest of the authors. In particular, we are interested in the assessment, evolution and potential prediction of algal blooms that have the potential to include harmful algal species (i.e., Harmful Algal Blooms (HABs)). The environmental triggers leading to the onset, evolution and dissemination of $\mathrm{HAB}$ events are widely unknown and are under active investigation.

Given the ecological and socio-economic importance of coastal regions, like Southern California (U.S. Commission on Ocean Policy 2004), it is important to be able to accurately assess, and ultimately predict how changes driven by urbanization and climate impact these areas.

In addition to regional anthropogenic disturbances, Southern California experiences significant decadal and internannual variability associated with the Pacific Decadal Oscillation (PDO) and El Niño-Southern Oscillation (ENSO) (Dailey, Reish \& Anderson 1993) and (Kennedy, Twilley, Kleypas, J. H. Cowan \& Hare 2002). These climatic phenomena impact the frequency and intensity of the regional episodic storm events, as well as the physical and biogeochemical dynamics of the coastal marine ecosystem. The increased rainfall in an urban, coastal region results in freshening of sea-surface waters through direct rainfall into the ocean and from freshwater inflow at the coastal boundary from streams and rivers. The river runoff supplies nutrient-rich waters to the ocean surface, which may lead to a bloom of photosynthetic organisms (i.e., algal bloom).

An open question in coastal ocean science is to disseminate whether or not we can distinguish anthropogenically affected processes from natural variations and effects. The ability to track and monitor evolving features resulting from anthropogenic inputs can help answer this question and others related to the increased urbanization of coastal regions.

\subsection{Harmful Algal Blooms}

Microscopic organisms are the base of the food chain and are what all aquatic life ultimately depends upon for food. There are a few dozen species of phytoplankton and cyanobacteria that can create potent toxins when provided with the right conditions. These harmful algae can cause harm via toxin production, or by their accumulated biomass which may affect levels of dissolved oxygen in the water. Impacts to humans from HABs include, but are not limited to, severe illness and potential death following consumption of, or indirect exposure to HAB toxins. In addition, coastal communities and commercial fisheries can suffer severe economic losses due to fish, bird and mammal mortalities, and decrease in tourism due to beach closures. For these reasons, it is of interest to predict when and where HABs may form and which coastal areas they may affect. For general information on HABs, we refer the interested reader to (Anderson 2008). Harmful algal blooms are an active area on all coasts of the United States, and are of large concern for coastal communities in Southern California (Schnetzer, Miller, Schaffner, Stauffer, Jones, Weisberg, DiGiacomo, Berelson \& Caron 2007).

A related threat of HABs deals with the sinking of Pseudonitzschia, i.e., transfer of toxicity to the sea floor, as discussed in (Wood, Schnetzer, Benitez-Nelson, Anderson, Berelson, Burns, Caron, Ferry, Fitzpatrick, Jones, Miller, Morton \& Thunell 2009). Autonomous gliders have been used recently during the North Atlantic Bloom experiment to track the sinking of primary production from the surface to 800-1000 meters (Gray, Lee, D'Asaro, Perry, Fennel, Briggs, Rehm, Sackmann, Sieracki, Poulton \& Gudmundsson 2008). It is of interest to document the sinking of the pseudonitzschia biomass to determine the harmful effects away from the surface. Vertical movement or aggregation at specific depths can also be important factors affecting the abundance of some harmful algae such as dinoflagellates and their associated toxins (Kudela, Ryanb, Blakelya, Lanea \& Petersona 2008). Marine biologists are interested in defining the vertical zonation and migratory patterns of the dinoflagellate, and the interactions of the 


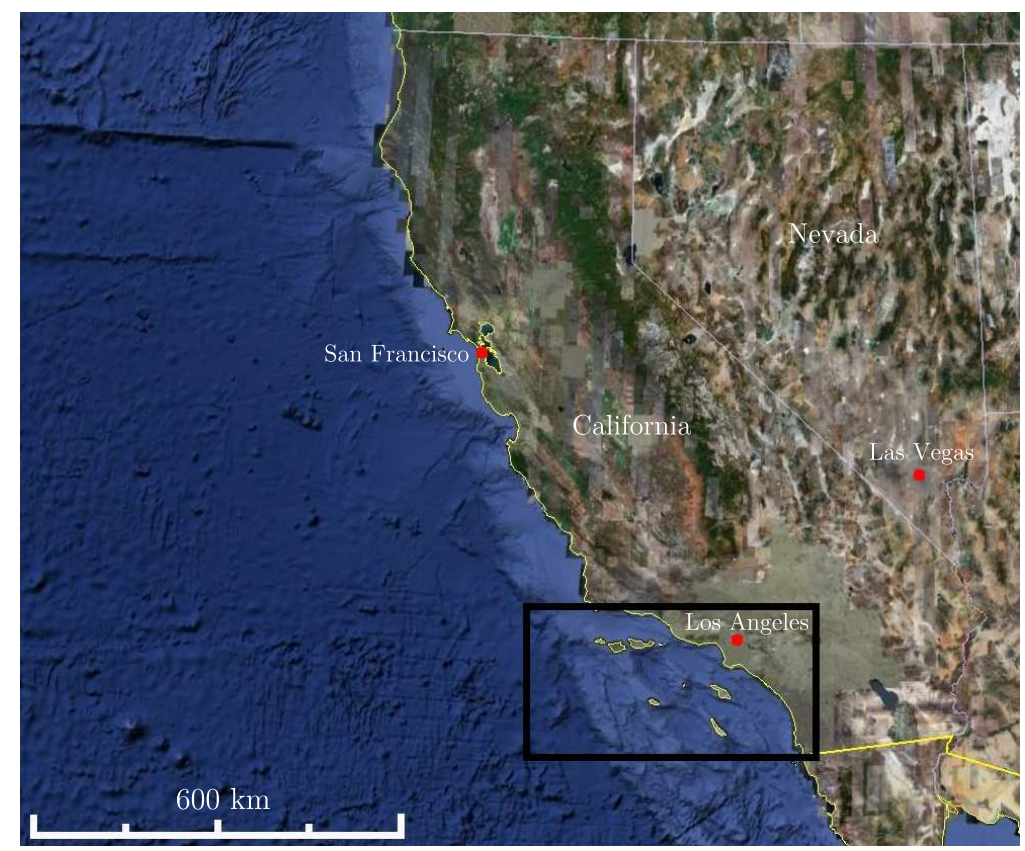

(a) California coastline. The Southern California Bight is the oceanic region contained in the black box, which is expanded in Fig. 1(b).

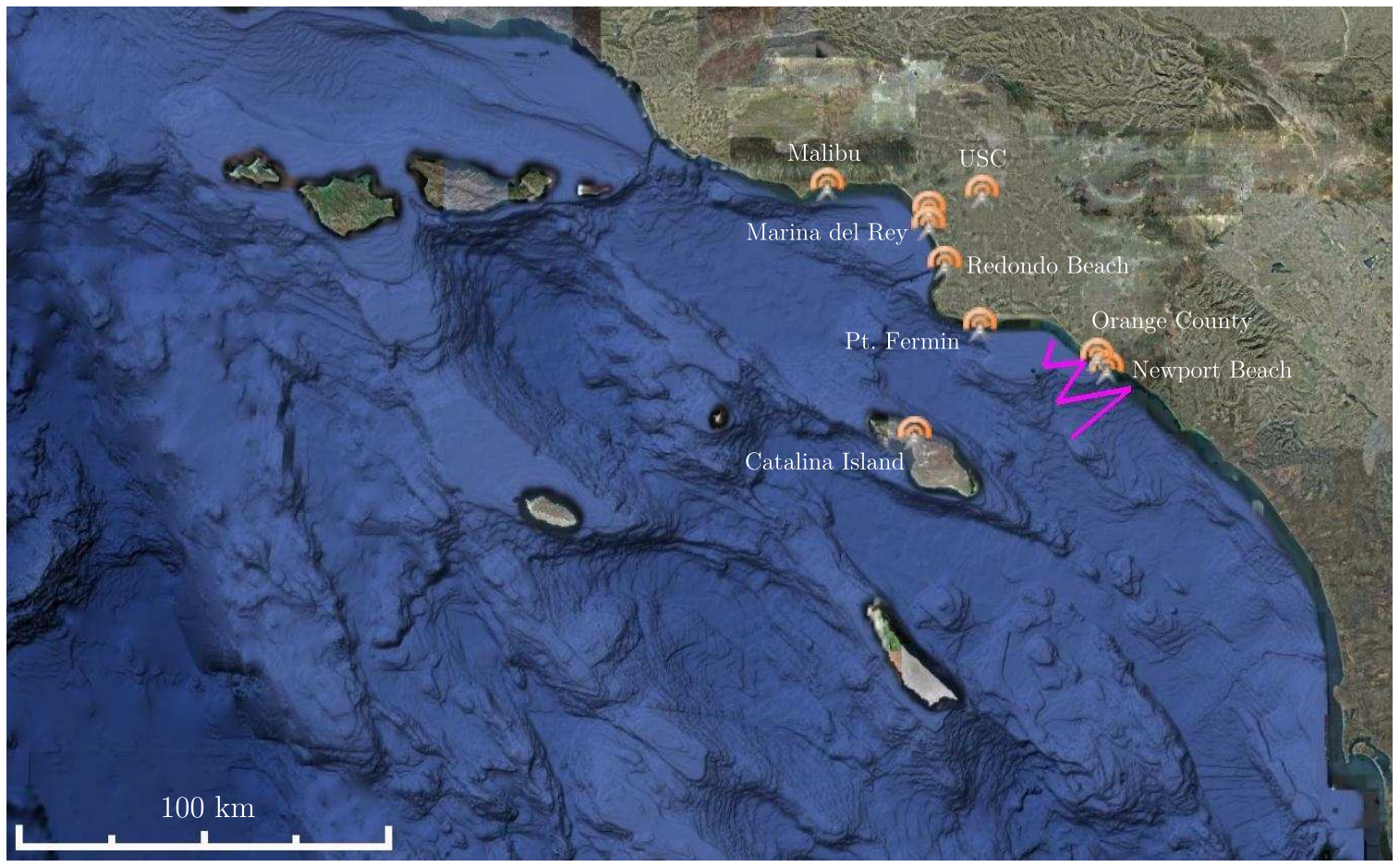

(b) The Southern California Bight.

Figure 1: The Southern California Bight (SCB). The primary area of interest, investigation and deployment. 
organism with physical processes of the ocean (e.g., currents, shear, density gradients, light) and the chemical structure (e.g., nutrients). Hence, with their unique sawtooth-shaped trajectories, ASGs are a good candidate platform to study surface blooms as well as their vertical migration.

Motivated by the number of problems linked to HABs, and algal blooms in general, it is of interest to study ocean features that can potentially promote a bloom event. In particular, blooms are likely to occur when nutrient-rich waters are brought to the surface. Ocean processes and features of interest promoting these conditions are cold-core eddys, upwelling, river runoff and waste water outfalls. These events alter the biochemical composition of the surrounding water, and provide the excess nutrients to support higher productivity and a bloom of microorganisms.

From the coastal dynamics and rapid urbanization in Southern California coastal communities discussed earlier, we choose plumes from river runoff and waste water outfalls as features to track and monitor. Both of these events can be discretely quantified; river runoff happens after a storm event and most waste water outfalls are human controlled. Hence, we have a good idea when and where a plume will be present in the SCB, and can employ the proper means to detect its onset and evolution.

The density of the considered plumes is less than the surrounding sea water, and forms a lens on the surface. The movements of these plumes are dominated by surface currents and local winds. The focus of this paper is on tracking the movement of plumes through the ocean by use of predictive tools and ASGs to study the factors and conditions leading to the onset and lifespan of a HAB event. We remark that similar techniques to those presented here can be applied to study eddys and upwelling, however with the choice taken here, we increase the likelihood of catching an actual event upon which to implement this innovative technology toolchain and trajectory design method.

\section{Regional Ocean Modeling System}

The predictive tool utilized in this study is the Regional Ocean Model System (ROMS) - a split-explicit, free-surface, topography-following-coordinate oceanic model. ROMS is an open-source, ocean model that is widely accepted and supported throughout the oceanographic and modeling communities. Additionally, the model was developed to study ocean processes along the western U.S. coast which is our primary area of study. The model solves the primitive equations using the Boussinesq and hydrostatic approximations in vertical sigma (i.e., topography following) and horizontal orthogonal curvilinear coordinates. ROMS uses innovative algorithms for advection, mixing, pressure gradient, vertical-mode coupling, time stepping, and parallel efficiency. Detailed information on ROMS can be found in (Shchepetkin \& McWilliams 1998) and (Shchepetkin \& McWilliams 2005).

The version of ROMS used in this study is compiled and run by the Jet Propulsion Laboratory (JPL), California Institute of Technology, and provides hindcasts, nowcasts and hourly forecasts (up to 36 hours) for the SCB via a web interface, (Vu 2008) or via access to their THREDDS Data Server (Jet Propulsion Laboratory 2009). The JPL version of ROMS (see e.g., (Chao, Li, Farrara, McWilliams, Bellingham, Capet, Chavez, Choi, Davis, Doyle, Frantaoni, Li, Marchesiello, Moline, Paduan \& Ramp 2008), (Li, Chao, McWilliams \& Ide 2008a) and (Li, Chao, McWilliams \& Ide 2008b)) assimilates HF radar surface current measurements, data from moorings, satellite data and any data available from sensor platforms located or operating within the model boundary.

This model utilizes a nested configuration, with increasing resolution covering the U.S. western coastal ocean at $15 \mathrm{~km}$, the Southern California coastal ocean at $5 \mathrm{~km}$, and the SCB at $1 \mathrm{~km}$. In addition to the $1 \mathrm{~km}$ output, a resampled $2.2 \mathrm{~km}$ resolution output, correlated to the assimilated HF radar grid resolution, is produced. The computations and predictions presented here use this $2.2 \mathrm{~km}$ resolution product.

The interaction with JPL related to this research and ROMS improvement is a two-way street. We need the predictions to design efficient, effective and innovative AUV trajectories. The JPL updates their ROMS by utilizing the feedback from field deployments to assess the validity of each prediction, and to increase the skill of future predictions.

\section{Mobile Sensor Platform: AUV}

The mobile sensor platforms used in this study are Webb Slocum autonomous underwater gliders (Webb Research Corporation 2008), see Fig. 2. An ASG is a type of AUV designed for long-term ocean sampling and monitoring (Schofield, Kohut, Aragon, Creed, Graver, Haldman, Kerfoot, Roarty, Jones, Webb \& 


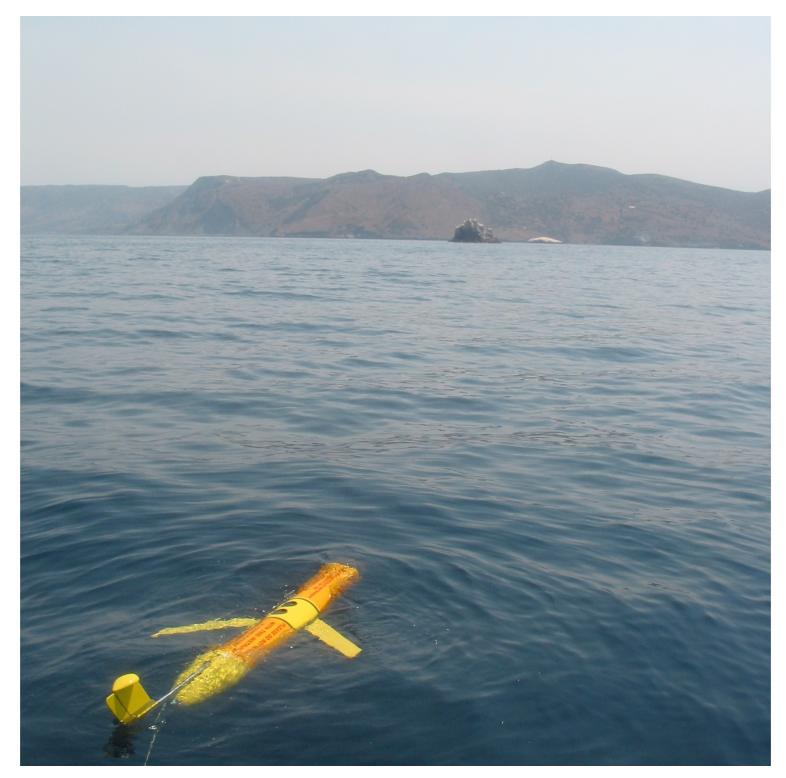

Figure 2: He Ha Pe, one of two USC Slocum gliders, preparing to begin a mission off the coast of Santa Catalina Island.

Glenn 2007). These gliders fly through the water by altering the position of their center of mass and changing their buoyancy. Due to this method of locomotion, gliders are not fast moving AUVs, have operational velocities on the same order of magnitude as oceanic currents $(\backsim 1 \mathrm{~km} / \mathrm{hr}$ ), and follow a sawtooth-shaped trajectory. The endurance ( $\sim 1$ month per deployment) and velocity characteristics of an ASG make it a good candidate vehicle to track plumes that move with ocean currents, and that have a residence time on the order of weeks. We have upgraded the communication capabilities of our gliders to take advantage of, and become a node in our local wireless network; details can be found in, (Pereira, Heidarsson, Caron, Jones \& Sukhatme 2009) and (Smith, Das, Heidarsson, Pereira, Caron, Jones \& Sukhatme 2009b).

Considerable work has been done on the kinematic and dynamic modeling and control of underwater gliders, and we refer the interested reader to (Leonard \& Graver 2001), (Graver 2005) and the references therein, for a detailed treatment of these topics. Here, we assume that the glider can successfully navigate from one location to another. This is a non-trivial assumption due to the complexity of the underwater environment and the forces experienced by an AUV while underway. An entire body of research exists that is dedicated to accurate and precise execution of prescribed missions by AUVs, and is outside the scope of the work presented here. In relation to this work, research is active to improve the accuracy of our glider trajectories by use of ROMS predictions in the SCB (Smith, Pereira, Chao, Li, Caron, Jones \& Sukhatme 2010).

Briefly, an example mission for a standard ASG consists of a set maximum depth along with an ordered list of geographical waypoints. An exact path or trajectory connecting these locations is not prescribed by the operator, nor are the controls to realize the final destination. When navigating to a new waypoint, an ASG computes a bearing and distance from its current location, then dead reckons (openloop control implementation) toward the goal location. While underwater, the computed bearing is not altered, the ASG must surface to make any corrections or modifications to the planned trajectory. Upon surfacing and acquiring a GPS fix, the positional error between actual and goal locations is computed. This error is used to estimate any local disturbances (e.g., currents), and is considered when computing the heading and bearing to the next waypoint.

\section{Problem Outline and Trajectory Design}

In this section, we formally pose the path planning problem and present the algorithms that generate the locations (waypoints) for the AUV to visit, which steer it to follow the general movements of a plume. Depending upon the feature considered, different locations within that feature may be of interest, e.g., 
its boundary or extent, subsurface chlorophyll maximum, salinity minimum, its centroid, etc. Here, we extend the work presented in (Smith et al. 2009a) to include the use of multiple vehicles to track different areas within an evolving plume. In particular, we consider the centroid and the boundary of the extent of a plume as two proxy areas of interest, and determine trajectories for multiple vehicles to track these locations within the plume. Similar algorithms to those presented here are able to consider alternate sampling locations and areas of interest.

Considerable study has been reported on adaptive control of single gliders and coordinated multiglider systems, see e.g., (Paley et al. 2007), (Paley et al. 2008) and the included references. In these papers, the trajectories given to the gliders were fixed patterns (rounded polygons) that were predetermined by a human operator. The adaptive control component was implemented to keep the gliders in an optimal position, relative to the other gliders following the same trajectory. The difference between the method used in (Paley et al. 2008) and the approach described here, is that our sampling trajectory is determined from the output of ROMS, and thus, at first glance, may appear as a seemingly random and irregular sampling pattern. Such an approach is a benefit to the model and scientist alike. Scientists can identify sampling locations based upon ocean measurements they are interested in following, rather than setting a predetermined trajectory and hoping the feature enters the transect while the AUV is sampling. When deploying multiple vehicles, this method allows the operator to generate trajectories that survey an appropriate spatial extent of the feature of interest. And, model skill is increased by the continuous assimilation of the in situ collected data; which by choice, is not a continuous measurement at the same location.

A plume may dissipate rapidly, but can stay cohesive and detectable for up to weeks. It is of interest to track these plumes based on the discussion in Sect. 2 as well as in (Cetinic, Jones, Moline \& Schofield 2008). In addition to tracking the plume, it is also important to accurately predict where a plume will travel on a daily basis. The ROMS prediction capabilities are good, but model skill can significantly increase from assimilation of in situ measurements.

\subsection{Problem Statement}

Given a freshwater plume, we are interested in designing trajectories to guide ASGs to track and sample along the path of the centroid, as well as the boundary. We assume that we have at least two vehicles to perform the missions; one centroid tracker and one boundary tracker. Due to the large amounts of chromophoric dissolved organic matter, a plume resulting from river runoff or waste water outfall can easily be identified from satellite imagery. Additionally, these directly follow a rain event, and the discharge location (i.e., river mouth) is well-known or, in the case of waste water outfalls, is determined by the local sanitation district. Thus, we may assume that we are aware of the occurrence and can delineate the extent of a plume at an initial point in time.

Since the propagation of the plume is determined by local winds and surface currents, we can forecast its hourly movement by use of ROMS surface current predictions, see (Smith et al. 2009a). The prediction begins with the initial delineation of the plume and is basis for determining the waypoints that define the trajectory. For safety concerns, we restrict an ASG to surface no more than once every four hours. In (Smith et al. 2009a), we considered surface intervals as short as one hour. However, surfacing that frequently kept the vehicle close to the surface and in danger of collision with other vessels ${ }^{2}$. Additionally, upon surfacing the ASG acquires a GPS fix, communicates its position and possibly collected data to the network. This communication time is significant ( $\sim 15 \mathrm{~min})$ when considering the temporal aspect of tracking a moving plume. This gives further support for the restriction to a four hour interval between surfacings. Since the basic idea is to track the plume for many days, while assimilating collected data into the model, the accuracy of the model prediction degrades with time, and we need time to run the model each day, we choose to plan a $T=16$ hour tracking and sampling mission for each day as early in the ROMS prediction as possible.

The starting location $L$ of each vehicle is known, and we assume that the prediction of the plume evolution is accurate. The initial delineation of the plume is done by selecting a set of geographic locations that encompass the plume's extent. These discrete locations are then forecasted as if they were Lagrangian drifters in the ROMS prediction. Additionally, we assume that the glider travels at a constant speed $v \mathrm{~km} / \mathrm{h}$, and $d_{h} \mathrm{~km}$ is the distance traveled in $h$ hours. There is no adaptive behavior

\footnotetext{
${ }^{2}$ The SCB is a very high traffic region, and the glider has a low visual profile when on the surface. Although the probability of a collision is low, we choose to err on the side of caution.
} 
incorporated during the execution of the planned trajectory. The idea is to improve the collection of scientific data by predicting the best locations to send an ASG, while also providing feedback to JPL on the accuracy of ROMS. In the long run, both communities will benefit.

\subsection{Waypoint Selection Algorithms}

In this section, we present the centroid and boundary-tracking, waypoint-generation algorithms. These algorithms utilize the ROMS prediction of a delineated plume to generate a sampling mission that guides the AUV to locations of interest or scientific merit within the given feature. Note that we neither consider vehicle dynamics nor the effect of ocean currents upon the vehicle in this study; these are areas of ongoing research. Also, we only consider the 2-D planar problem plus time, as far as the waypoint computation is concerned.

\subsubsection{Centroid-Tracking Algorithm}

We begin with the centroid-tracking, waypoint-generation algorithm. The input to the trajectory design algorithm is a set of points, $\mathcal{D}$ (referred to as drifters) that determine the initial extent of the plume $\left(\mathcal{D}_{0}\right)$, and hourly predictions $\left(\mathcal{D}_{i}, i \in T\right)$ of the location of each point in $\mathcal{D}$. For the points in $\mathcal{D}_{i}$, we compute the convex hull as the minimum bounding ellipsoid, $E_{i}$ for $i \in T$. We consider the predicted locations of $\mathcal{D}_{0}$ after four hours, $\mathcal{D}_{4}$. The centroid of $\mathcal{D}_{4}$ is $C_{4}$ (the centroid of $E_{4}$ ). The algorithm computes $d_{g}\left(L, C_{4}\right)^{3}$. Given upper and lower bounds $d_{u}$ and $d_{l}$, respectively, we have three possible cases. Case 1: If $d_{l}<d_{g}\left(L, C_{4}\right) \leq d_{u}$, the generated waypoint is $C_{4}$, and the trajectory is simply defined as the line $\overline{L C_{4}}$, see Fig. 3(a). Case 2: If $d_{g}\left(L, C_{4}\right) \leq d_{l}$, the algorithm first checks to see if there exists a point $p \in E_{4} \cup \mathcal{D}_{4}$ such that

$$
d_{l} \leq d_{g}(L, p)+d_{g}\left(C_{4}, p\right) \leq d_{u} .
$$

If such a point exists, the algorithm generates two waypoints ( $p$ and $\left.C_{4}\right)$ and the trajectory is defined as the line $\overline{L p}$ followed by the line $\overline{p C_{4}}$. In general, this will not be the case, since the distance from the centroid of the plume to its boundary is many kilometers. Thus, if $\left\{p \in E_{4} \cup \mathcal{D}_{4} \mid d_{l} \leq d_{g}(L, p)+d_{g}\left(C_{4}, p\right) \leq\right.$ $\left.d_{u}\right\}=\emptyset$, then the algorithm computes the locus of points, $\mathcal{L}=\left\{p^{*} \in \mathcal{L} \mid d_{g}(L, p)+d_{g}\left(p, C_{4}\right)=d_{4}\right\}$ and selects a point at random $p^{*} \in \mathcal{L}$ as another waypoint. Here, the trajectory is the line $\overline{L p^{*}}$ followed by the line $\overline{p^{*} C_{4}}$, see Fig. 3(b). This additional waypoint computation was inserted into the algorithm when considering a single vehicle deployment. In this scenario, one would like to acquire as much data as possible. In the case of a multiple vehicle mission, it is less useful to include the additional waypoint in the trajectory design, as the other vehicles are gathering supplemental data. During deployment, $p^{*}$ is visited only if we feel the safety of the vehicle will not be compromised by frequent surfacings (e.g., based on geographical location, day of the week and time of day). Case 3: If $d_{g}\left(L, C_{4}\right)>d_{u}$, the algorithm generates a waypoint $C_{w}$ in the direction of $C_{6}$, such that $d_{g}\left(L, C_{w}\right)=d_{4}$, see Fig. 3(c). Let $A Z(a, b)$ be the azimuth angle between locations $a$ and $b$. The location of the vehicle $L$ is updated to $C_{4}$ or $C_{w}$ and the process is iterated for duration $T$. This waypoint generation process is presented in Algorithm 1.

\subsubsection{Boundary-Tracking Algorithm}

Similarly to the presentation in Sect. 5.2.1, we define the boundary-tracking, waypoint-generation algorithm, presented in Algorithm 2. We begin with the same predictions as above, and define $P_{i}$ to be the polygon formed by connecting the points $\mathcal{D}_{i}$ for $i \in T$. Let $B(p, r)$ be the 2-D disc of radius $r$, about $p$. This algorithm first computes $N=B\left(L, d_{4}\right) \cap P_{4}$. Again we have three possible cases to investigate. Case 1: If $N \geq 2$, the generated waypoint $B_{4}$ is a random selection of one of the intersection points, see Fig. 4(a). Case 2: If $N=1$, the generated waypoint $B_{4}$ is that precise intersection point, see Fig. 4(b). Case 3: If $N=\emptyset, B_{4}$ is computed such that $d_{g}\left(L, B_{4}\right)=d_{4}$ and $A Z\left(L, B_{4}\right)$ is the average azimuth of $\mathcal{D}_{i}$ for the considered four hour time period, see Fig. $4(\mathrm{c})$. We reassign $L=B_{4}$, and the algorithm is repeated. For the boundary tracking scenario, it would be of interest to traverse the entire predicted extent of the given plume over the duration of the survey. However, the test-bed vehicles considered move too slowly to entertain this sampling method. Thus, we choose to select a random point on the boundary when posed with multiple options. Also, since we are interested in assessing the dispersion,

\footnotetext{
${ }^{3}$ We define $d_{g}(x, y)$ to be the geographic distance from $x$ to $y$
} 


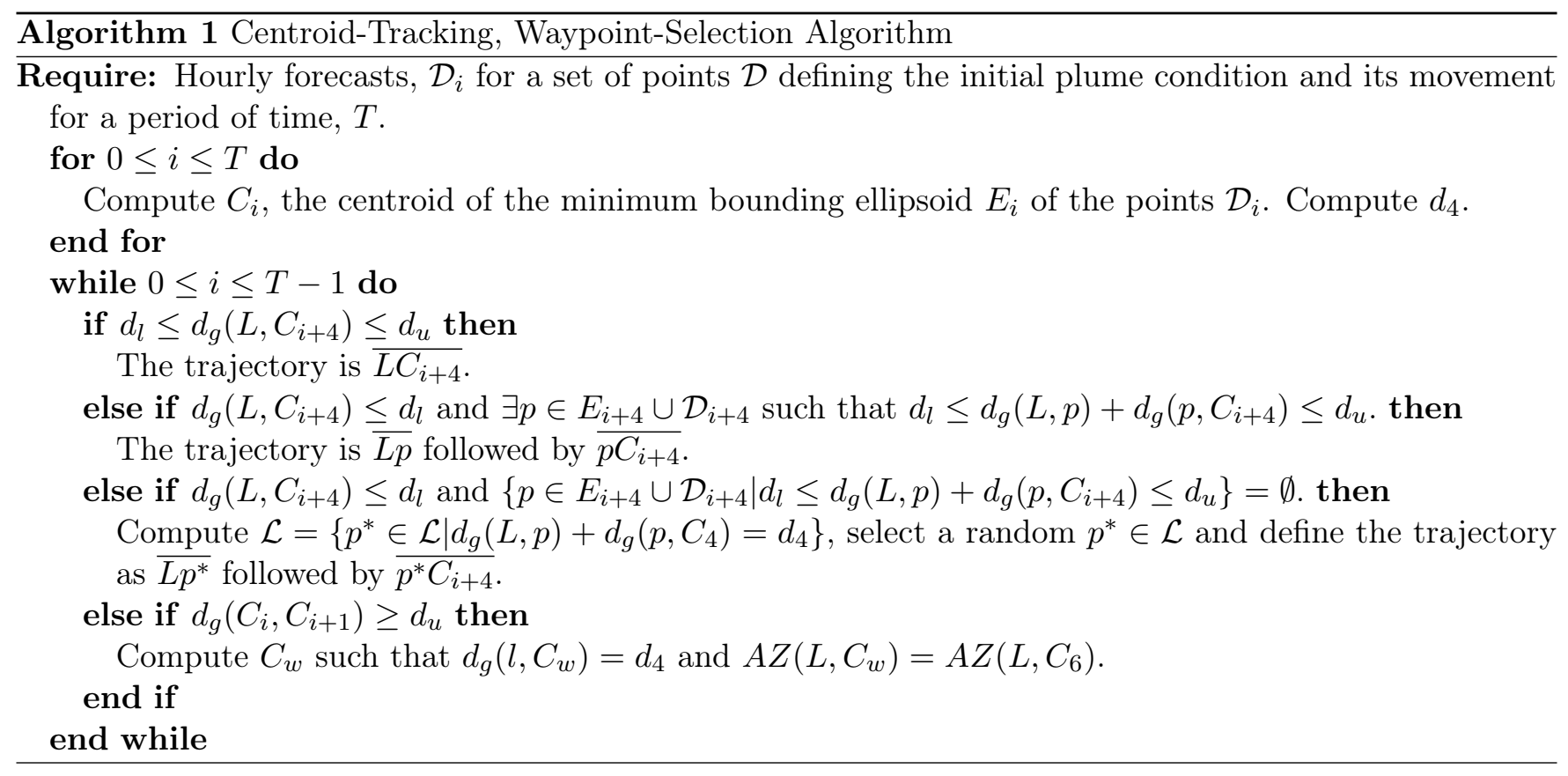

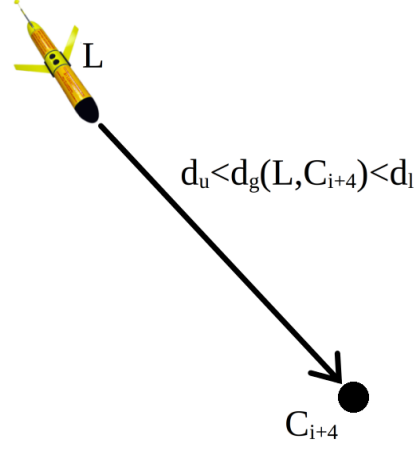

(a) Case 1

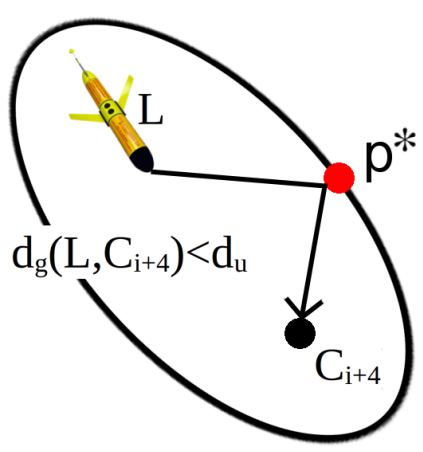

(b) Case 2

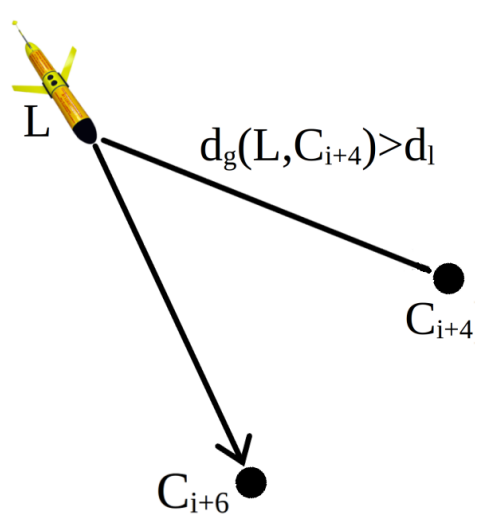

(c) Case 3

Figure 3: The three possible cases considered for the centroid tracking algorithm. 


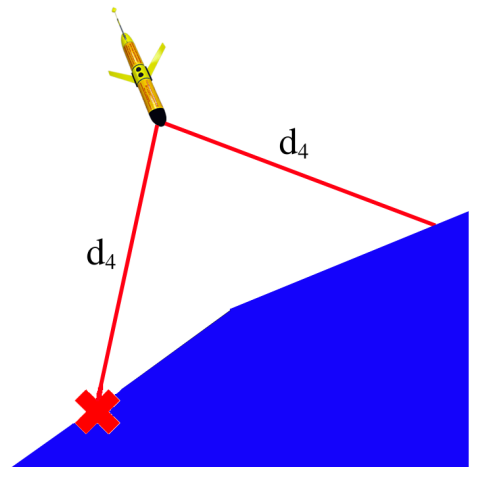

(a) Case 1

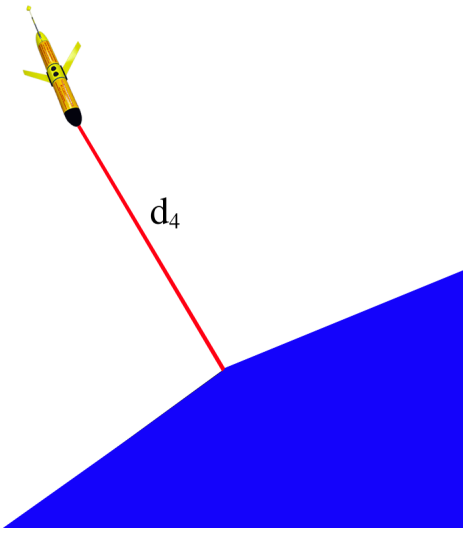

(b) Case 2

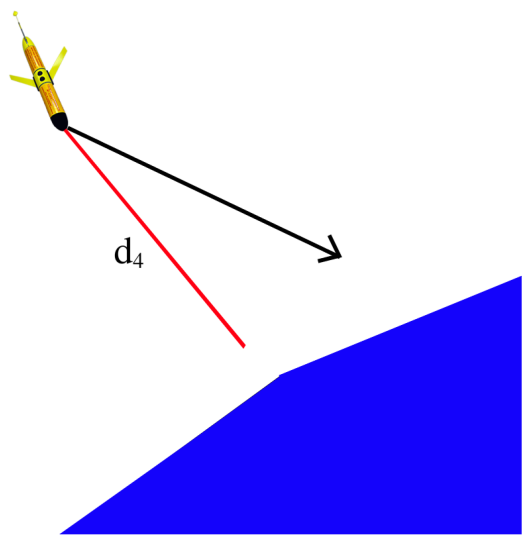

(c) Case 3

Figure 4: The three possible cases considered for the boundary tracking algorithm.

and potentially the subsurface mixing of the plume waters with the surrounding ocean waters, navigating near or criss-crossing the actual plume boundary can gather interesting data.

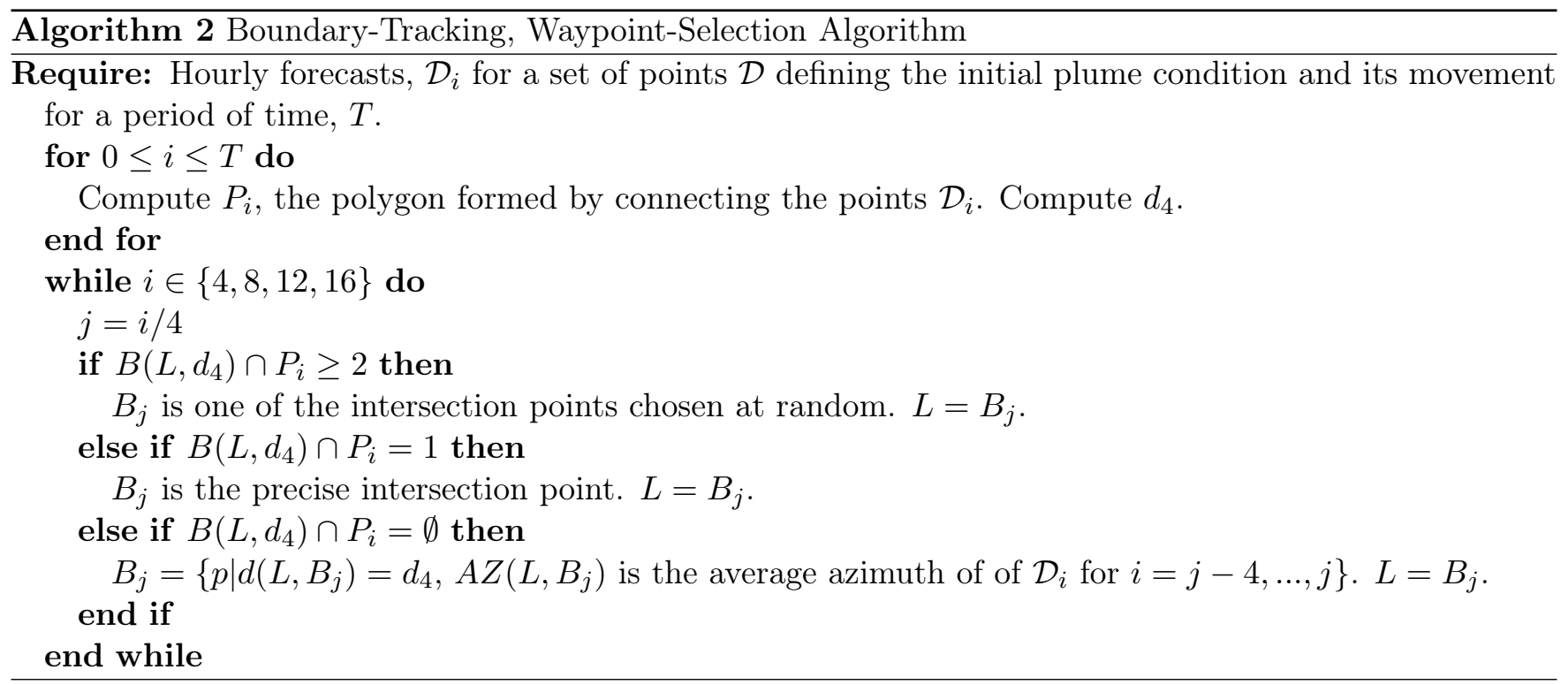

\subsubsection{Ocean Plume Tracking Algorithm Based on Ocean Model Predictions}

After we have generated the waypoints that define the trajectory for the vehicle to follow, we implement an iterative procedure to track the feature of interest over multiple days. This involves assimilating gathered data into ROMS and updating the projections for generating the trajectories for subsequent days. This overall iterative process to design an implementable plume tracking strategy based on ocean model predictions is given in Algorithm 3.

REMARK 5.1 In the SCB, a vertical velocity profile of ocean current is, in general, not constant. Since the plume propagates on the ocean surface $(1-3 \mathrm{~m})$ and the glider operates from the surface down to depths of $60-100 \mathrm{~m}$, it is not valid to assume that they are subjected to the same current regime, in both velocity and direction. Thus, it may be possible for a plume to outrun a slow-moving vehicle (i.e., $d_{g}\left(C_{i}, C_{i+1}\right) \geq d_{u}$ or $\left.N=\emptyset\right)$. For emphasis, a representative current profile, of the meridional velocity with respect to depth, is given in Fig. 5. 

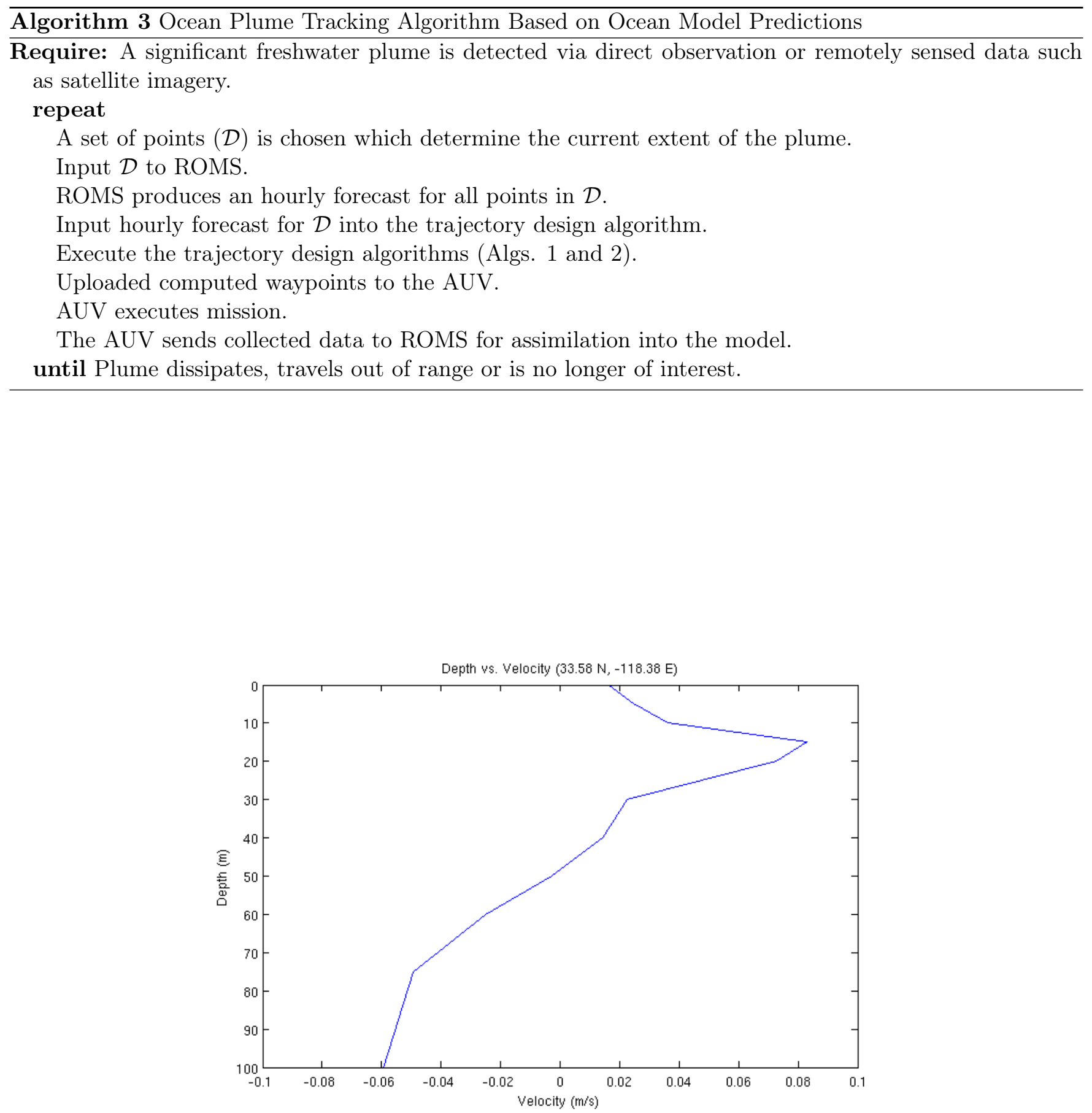

Figure 5: Ocean depth versus current velocity for the meridional component of the ROMS predicted flow at $33.58^{\circ} \mathrm{N},-118.38^{\circ} \mathrm{E}$. 


\section{Implementation and Field Experiments in the SCB}

We present the results of two field deployments that implemented trajectories designed by Alg. 3. In Sect. 6.1 we present the results of a single vehicle, centroid-tracking mission initially presented in (Smith et al. 2009a). We follow this in Sect. 6.2 with a two vehicle mission, tracking both the centroid and boundary of a plume.

As noted in (Smith et al. 2009a), the rainy season in Southern California is generally between November and March. During this time, storm events cause large runoff into local area rivers and streams, all of which empty into the Pacific Ocean. Two major rivers in the Los Angeles area, the Santa Ana and the Los Angeles River, input large freshwater plumes to the SCB. Such plumes have a high likelihood of producing HABs. Unfortunately, during both deployments, weather and/or remote sensing devices did not cooperate to produce a rain event along with a detectable plume. For both cases presented here, we defined a pseudo-plume in two separate areas of the SCB to demonstrate a proof-of-concept of the technology chain and trajectory design method developed here.

For the centroid tracking mission, we deployed an ASG into the SCB on February 17, 2009 to conduct a month-long observation and sampling mission. For this deployment, the glider was programmed to execute a zig-zag pattern mission along the coastline, as depicted in Fig. 7, by navigating to each of the six waypoints depicted by the red and black bulls-eyes. Figure 7 also delineates the $20 \mathrm{~m}$ and $30 \mathrm{~m}$ isobaths, given by the green and red lines, respectively. During execution of this mission, we retasked the ASG mid-mission and uploaded the centroid-tracking trajectory described in Sect. 6.1.

For the boundary tracking mission, we deployed two ASGs off of the northeast tip of Santa Catalina Island on April 29, 2009 to conduct a month-long experiment to test the communication infrastructure described in (Smith et al. 2009c). For this mission, there was not a single, pre-determined path for the glider to traverse as before, but we had the ability to retask the vehicles as needed. The details of this mission, with regard to the communication data collected, can be found in (Pereira et al. 2009) and (Smith et al. 2009b). The two day mission presented below was conducted during May 11-13, 2009.

During deployment, in addition to validating the trajectory design and innovative toolchain presented here, the glider(s) also gather oceanographic data that is both assimilated into ROMS and processed for assessment and analysis by members of the CINAPS team. The USC ASGs collect temperature, salinity, depth and chlorophyll-a, and will soon be fitted with nutrient sensors to measure phosphate and nitrate levels as well. A 3-D interpretation of data collected along the San Pedro shelf, during a deployment that occurred in early April 2009, is presented in Fig. 6. This figure displays the measured temperature along the glider's path between two surface intervals.

\subsection{Centroid Tracking}

The mission presented in this section is reproduced from (Smith et al. 2009a). Since Alg. 1 has been modified based on the lessons learned during the execution of this deployment, there are slight discrepancies in planning between the following description and the method presented in Alg. 1. However, the general idea and method is the same.

For this mission, we defined a pseudo-plume $\mathcal{D}$ with 15 initial drifter locations off the coast of Newport Beach, CA. The pseudo-plume is given by the blue line in Fig. 8 .

By use of ROMS, the locations of the points in $\mathcal{D}$ were predicted for $T=15$ hours. The initial time and location for the beginning of this retasking experiment coincided with predicted coordinates of a future glider communication. The pseudo-plume was chosen such that $C_{0}$ was near this predicted glider surfacing location.

Based on observed behavior for our vehicle during this deployment, we take $v=0.75 \mathrm{~km} / \mathrm{h}$, and initially defined $d_{l}=0.5 \mathrm{~km}$ and $d_{u}=0.8 \mathrm{~km}$. The hourly predictions were input to the trajectory design algorithm and a tracking strategy was generated. Due to slow projected surface currents in the area of study, the relative movement of the plume was quite small. To keep the glider from surfacing too often and to generate a more implementable trajectory, we opted to omit visiting consecutive centroids. Instead, we chose to begin at the initial centroid, then visit the predicted centroid of the plume after five, ten and 15 hours, $C_{5}, C_{10}$ and $C_{15}$, respectively. Between visiting these sites, the algorithm computed an additional waypoint for the glider to visit. These intermediate waypoints were chosen similarly to the $p^{*}$ defined earlier, with $d=3.75 \mathrm{~km}$; the distance the glider should travel in five hours. This design strategy produced seven waypoints for the AUV to visit during the 15 hour mission. The waypoints are presented in Table 1. Note that we include the initial centroid as a waypoint, since the glider may not 


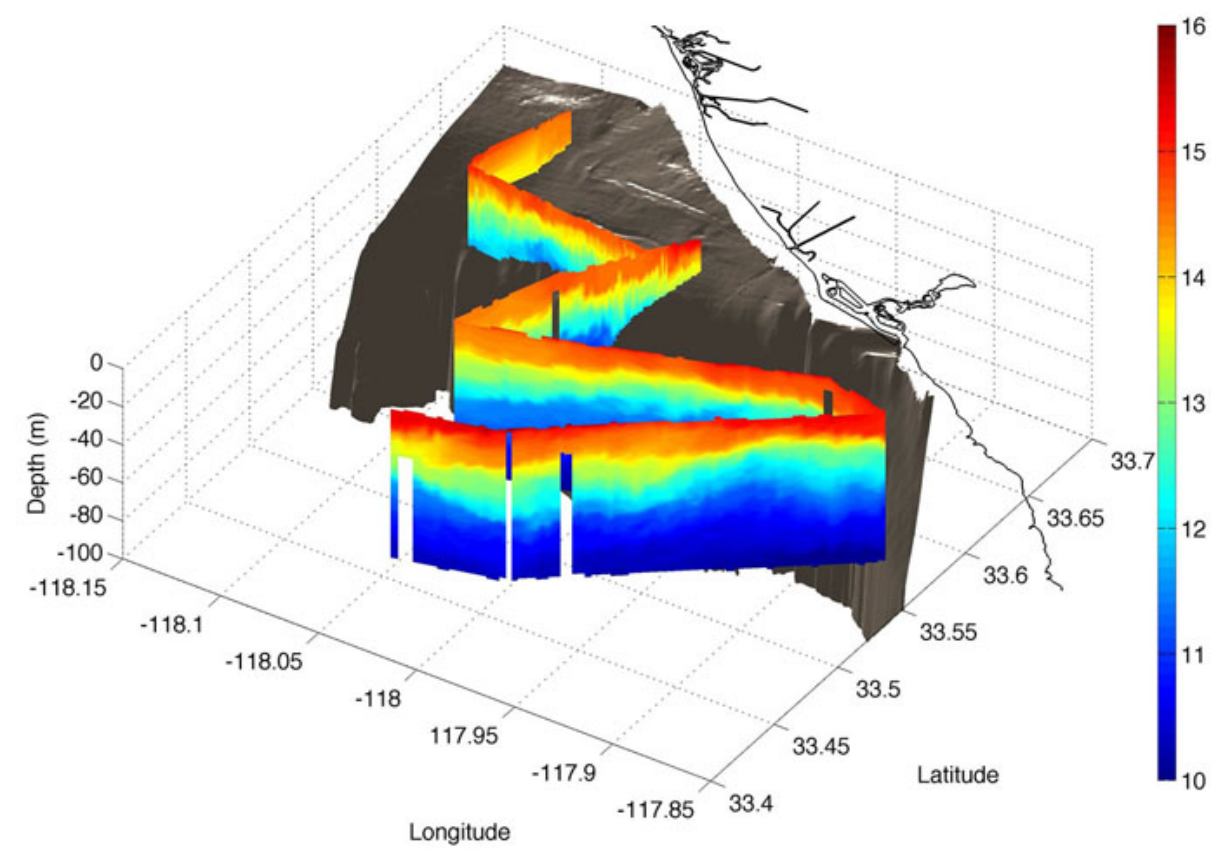

Figure 6: Three-dimensional depiction of temperature data collected along a glider trajectory during a deployment in April 2009. The scale bar to the left is in degrees Centigrade.

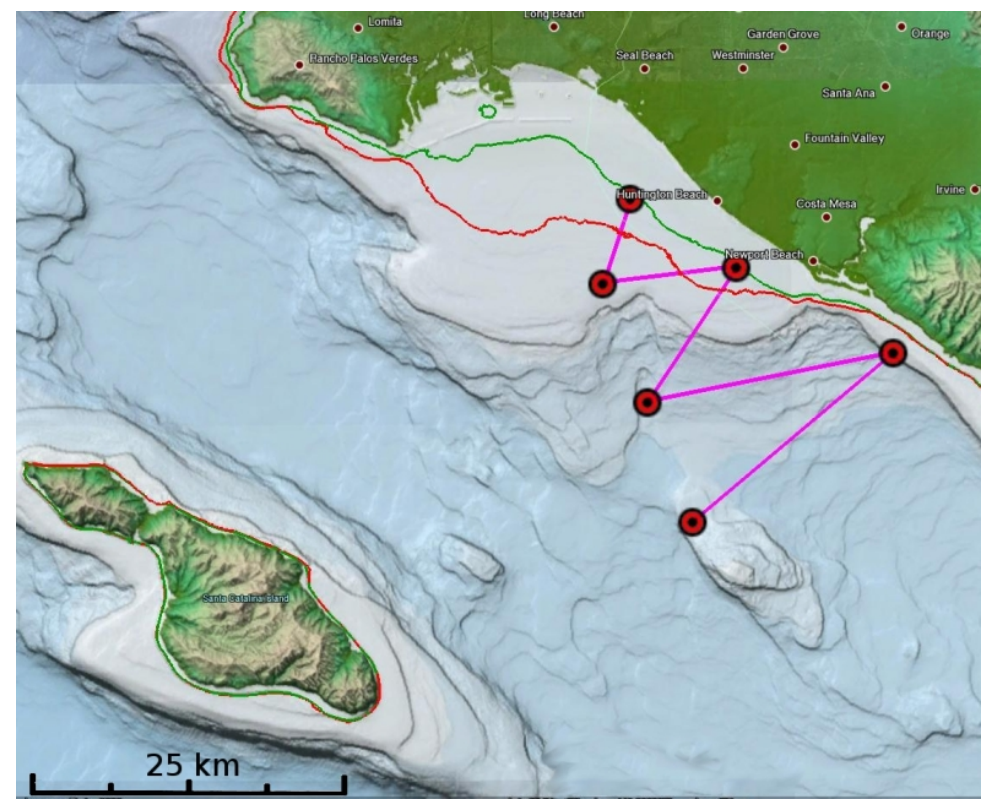

Figure 7: Preset waypoints, depicted with red and black bulls-eyes and the intended path of the glider given by the magenta line. Image created by use of Google Earth. 


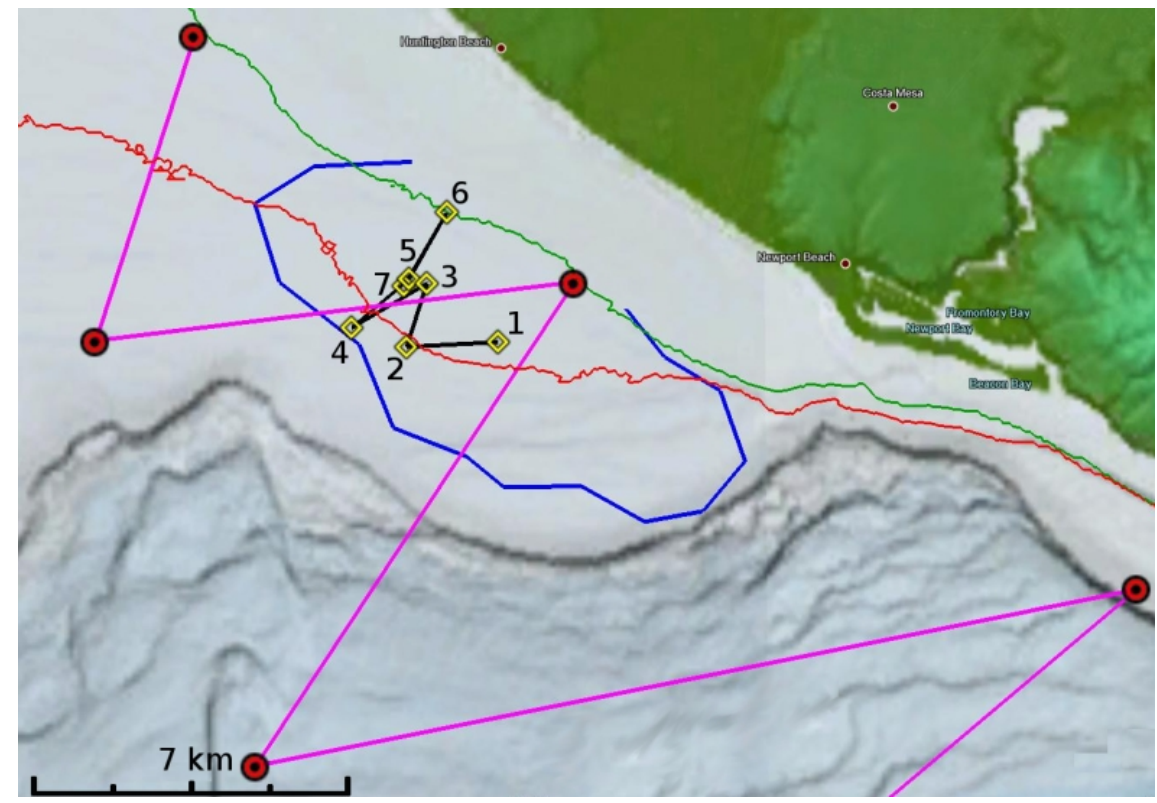

Figure 8: Plume (blue line), computed waypoints (yellow diamonds), and path connecting consecutive waypoints (black line). Image created by use of Google Earth.

\begin{tabular}{|ccc|ccc|}
\hline Number & Latitude (N) & Longitude (E) & Number & Latitude (N) & Longitude (E) \\
\hline 1 & 33.6062 & -118.0137 & 5 & 33.6189 & -118.0349 \\
2 & 33.6054 & -118.0356 & 6 & 33.6321 & -118.0257 \\
3 & 33.6180 & -118.0306 & 7 & 33.6175 & -118.0361 \\
4 & 33.6092 & -118.0487 & & & \\
\hline
\end{tabular}

Table 1: Waypoints generated by the plume tracking algorithm. Waypoint numbers 1,3,5 and 7 are the predicted centroids of the pseudo-plume at hours $0,5,10$ and 15 , respectively.

surface exactly at the predicted location.

Upon visiting all of the waypoints in Table 1, the glider was instructed to continue the sampling mission shown in Fig. 7. Figure 8 presents a broad overview of the waypoints in Table 1, along with a path connecting consecutive waypoints. The plume is delineated by the blue line and the waypoints are numbered and depicted by yellow diamonds. Note that the glider did not travel on the ocean surface during this experiment. As previously mentioned, between waypoints the glider submerges below a set depth and performs consecutive dives and ascents creating a sawtooth-shaped trajectory as its glide path.

\subsubsection{Analysis of Results}

Next, we present the implementation results of the aforementioned sampling mission onto an ASG operating in the SCB. The waypoints given in Table 1 were computed under the assumption that the mission would be loaded onto the glider at a specific time and approximate geographic location. The glider arrived and communicated at the correct time and location, however, communication was aborted before the plume tracking mission could be uploaded. We were able to establish a connection two hours later at a different location, and successfully upload the mission file; this location is the red droplet labeled 1 in Fig. 9. We opted to not visit waypoint 1 based on the location of the glider and to get the glider back on schedule to track the plume. Figures 9 and 10 present magnified images of Fig. 8, where computed waypoints are the yellow diamonds and the red droplets are the actual locations visited by the glider.

We were able to successfully generate a plan and retask a deployed glider to follow an ocean feature for 15 hours. It is clear from the data that consideration has to be made for glider dynamics and external 


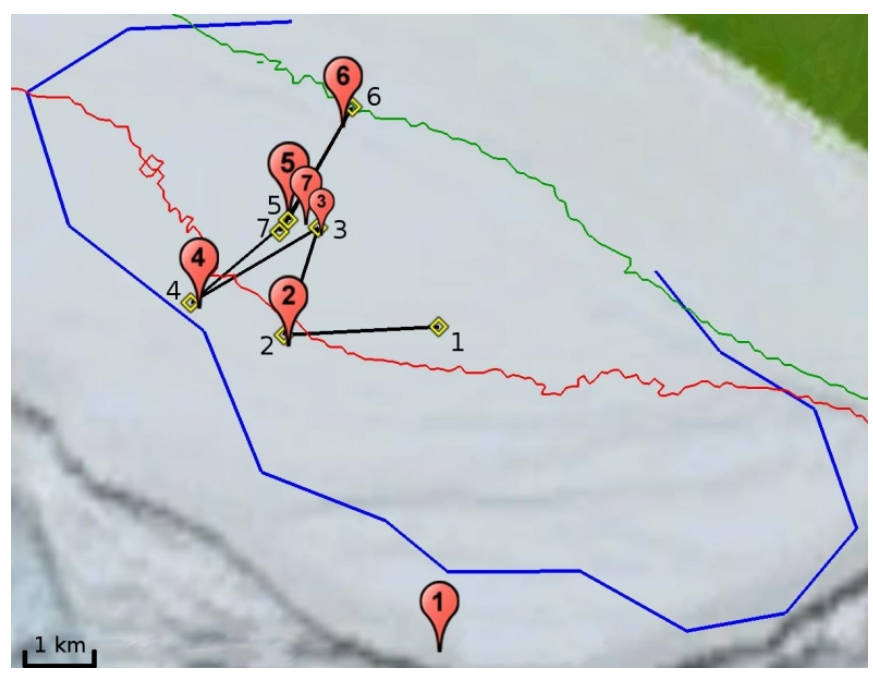

Figure 9: Computed waypoints (yellow diamonds) and actual glider locations (red droplets). Image created by use of Google Earth.

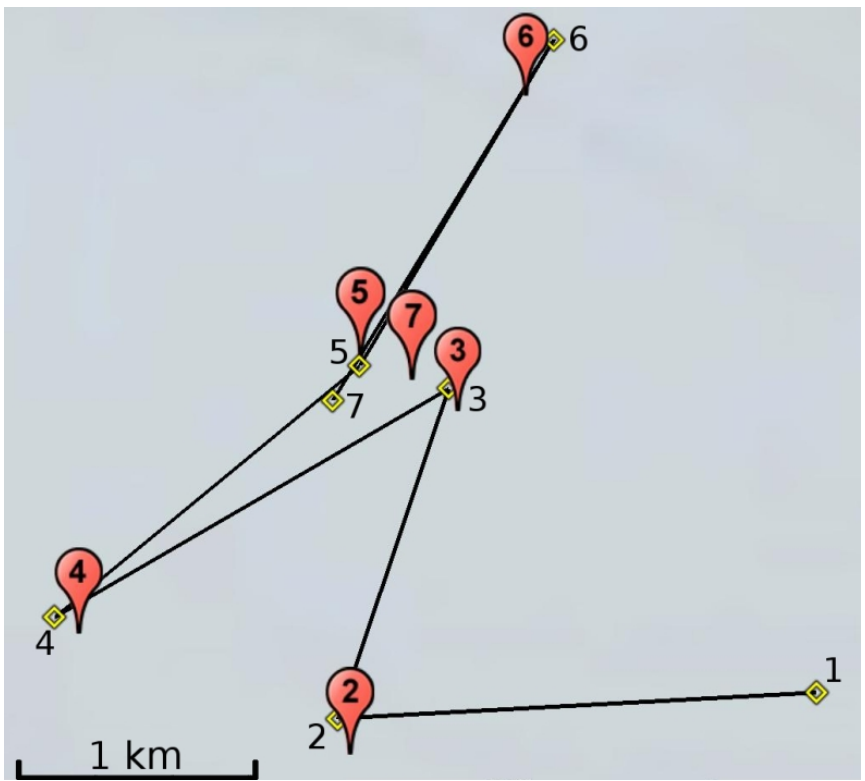

Figure 10: Computed waypoints (yellow diamonds) and actual glider locations (red droplets). Image created by use of Google Earth. 
forces from the ocean in the trajectory design algorithm. This is an area of active research.

One element that we have neglected to discuss up to this point is that we have no metric for comparison. In particular, when we reach a predicted centroid, we do not have a method to check whether or not the plume centroid was actually at that location. We are planning experiments to deploy actual Lagrangian drifters to simulate a plume, as well as hoping for an actual event to present itself. This will give a concrete comparison between the ROMS prediction and the actual movement of the drifters or the plume. Another component omitted from earlier discussion is time. When tracking a moving feature, a predicted waypoint contains temporal information as well as location. For this implementation, the glider began the mission at $0302 \mathrm{Z}$ and ended at 1835Z; a total time of 15.55 hours. Due to external disturbances, arrival at a few waypoints was not at the predicted time. The primary external disturbance affecting temporal, and spatial, accuracy is ocean currents. Preliminary efforts in the application of ocean current predictions in ASG path planning with regard to the problem studied in this paper can be found in (Smith et al. 2010).

\subsection{Centroid and Boundary Tracking}

For this mission, we defined a pseudo-plume $\mathcal{D}$ with 12 initial drifter locations off the northeast coast of Santa Catalina Island. Again, based on observed behavior, we take $v=0.75 \mathrm{~km} / \mathrm{h}$, and define $d_{l}=2.7$ $\mathrm{km}$ and $d_{u}=3.3 \mathrm{~km}$. The general testing area for this deployment is presented in Fig. 11(a). By use of ROMS, the locations of the points in $\mathcal{D}$ were predicted for $T=16$ hours. Due to the predicted currents and proximity to Catalina Island, two of the initially defined drifters exited the model boundary. Thus, the plume was propagated and computations were made by use of 10 drifters.

\subsubsection{Analysis of Results}

This retasking mission further validated the proof-of-concept of generating and implementing trajectories by use of predictive ocean models. In this case, we demonstrated further functionality by incorporating multiple vehicles and testing the iterative capabilities of the proposed technique by closing the loop with data assimilation into ROMS to update the next prediction. Since we used a pseudo-plume as a tracking proxy, we again do not have a metric in this experiment to assess any change in skill of ROMS predictions due to the data assimilation conducted. However, it is worth noting that collected data were successfully transmitted and assimilated from the robot in the field to ROMS for use in the next prediction. Using the methods described here to generate trajectories for multiple vehicles is not difficult. The complexity arises in synchronizing surfacings or compensating for asynchronous communication with the fleet of gliders. This synchronization problem was addressed in (Paley et al. 2008) for their specific application, and it is of interest to us to implement a similar, high-level control system to aid in the facilitation and management of multi-vehicle deployments incorporating autonomous retasking. Preliminary infrastructure and research toward this goal is presented in (Smith et al. 2009c) and (Pereira et al. 2009).

To begin, the plume was delineated in an area of current operation of two deployed ASGs. The initial location is presented in Fig. 11(b). The waypoints computed by Alg. 3 to follow this plume are presented for the centroid and boundary tracking vehicles in the left-hand columns of Tables 2 and 3, respectively. The initial time and location for the beginning of this retasking experiment coincided with predicted coordinates of a glider surfacings. Since the assigned mission was to collect communication data, the vehicles were surfacing frequently ( $\sim 2$ hour intervals). This made coordinating surfacing time much more manageable than in the scenario presented in Sect. 6.1, where the glider was surfacing approximately every eight hours. Additionally, communication via our implemented Freewave ${ }^{\mathrm{TM}}$ network (Pereira et al. 2009) facilitated more more robust communication as well as more rapid file exchange than during previous deployments. Once both gliders were on the surface together (approximately 1800Z, May 11, 2009), we ran Alg. 3 with the location of each glider initially being on the boundary of the plume. Figures 11(b) - 11(f) display the delineation of the plume with the locations of both gliders at four hour increments from $T=0$ hours (Fig. 11(b)) to $T=16$ hours (Fig. 11(f)). In these figures, the red glider is the boundary tracker and the yellow glider is the centroid tracker. The respective icons denote the locations where the glider surfaced while executing the mission. When visible, the orange dot represents the predicted centroid of the plume; when not seen, the yellow glider surfaced within $500 \mathrm{~m}$ of the location of the predicted centroid. The red and yellow lines depict the path of the glider (projected to the surface) between surfacings. Day one of this mission ended shortly after 1000Z on May 12, 2009. 


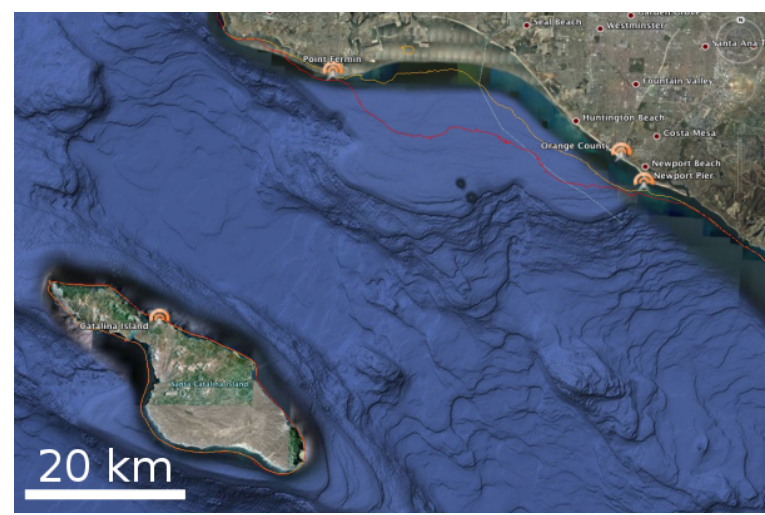

(a) Area Overview

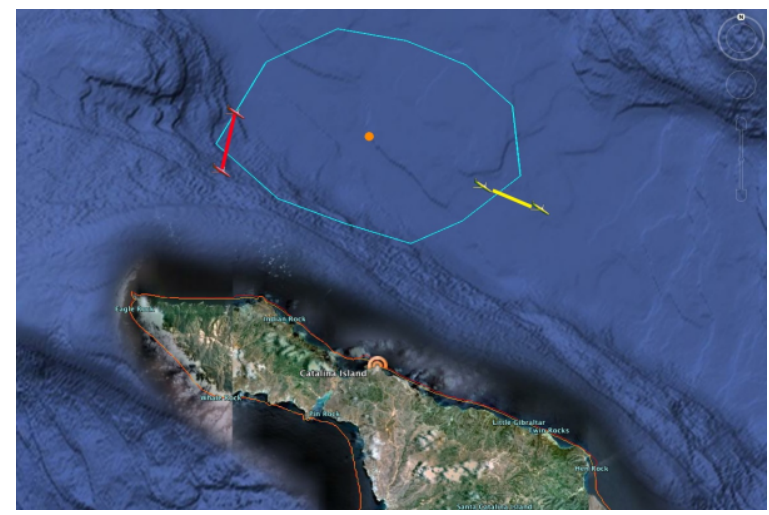

(c) $T=4 \mathrm{hrs}$

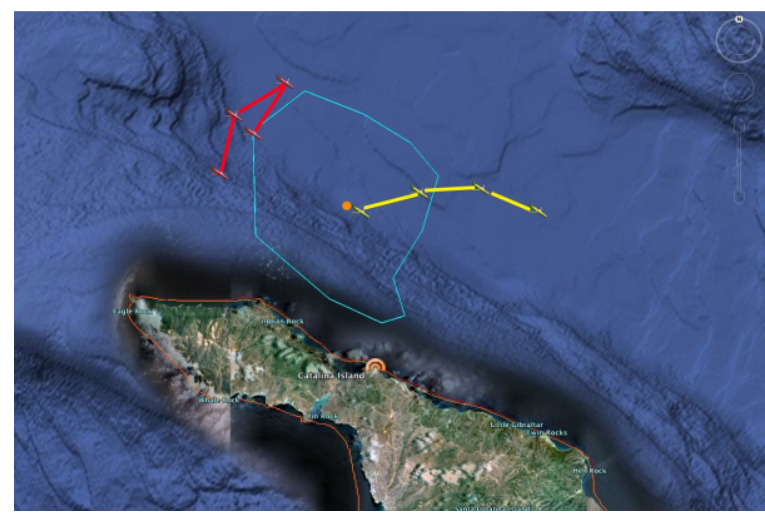

(e) $T=12 \mathrm{hrs}$

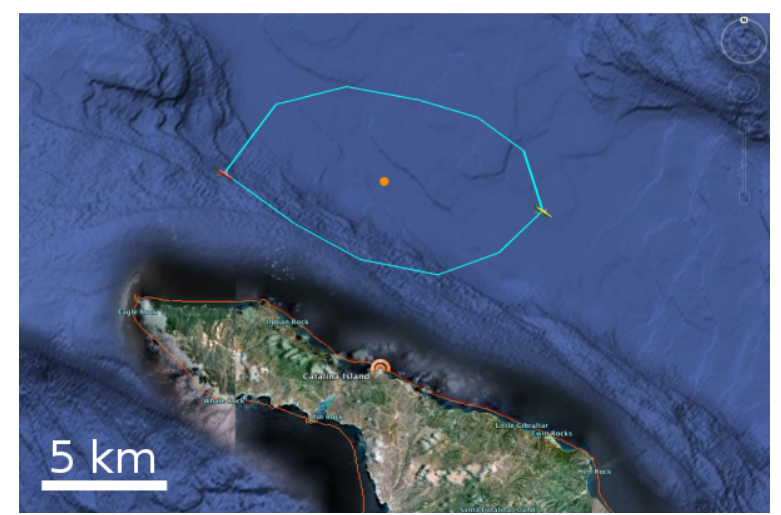

(b) $T=0 \mathrm{hrs}$

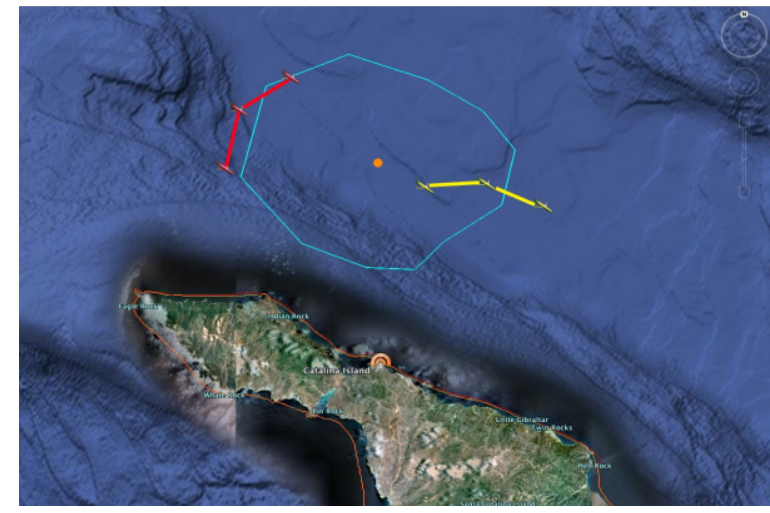

(d) $T=8 \mathrm{hrs}$

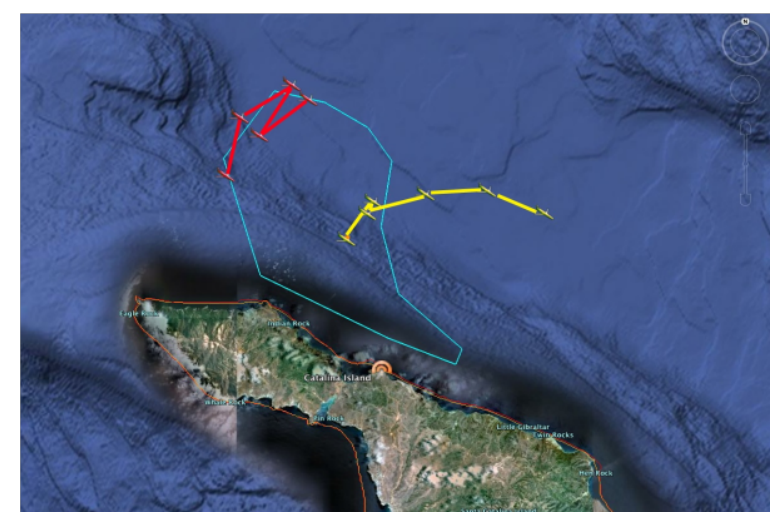

(f) $T=16 \mathrm{hrs}$

Figure 11: Centroid and boundary tracking experiment, Day 1. The scale shown in Fig. 11(b) is the same for Figs. 11(c) - 11(f) as well as Figs. 12(a) - 12(e). Images created by use of Google Earth. 


\begin{tabular}{|ccc|ccc|}
\hline Day 1 & Latitude (N) & Longitude (E) & Day 2 & Latitude (N) & Longitude (E) \\
\hline 1 & 33.5180 & -118.3930 & 1 & 33.5060 & -118.4970 \\
2 & 33.5281 & -118.4230 & 2 & 33.5236 & -118.4810 \\
3 & 33.5266 & -118.4553 & 3 & 33.5241 & -118.4901 \\
4 & 33.5177 & -118.4859 & 4 & 33.5060 & -118.4781 \\
5 & 33.5232 & -118.4826 & 5 & 33.5118 & -118.4809 \\
6 & 33.5061 & -118.4968 & 6 & 33.4867 & -118.4688 \\
& & & 7 & 33.4608 & -118.4523 \\
\hline
\end{tabular}

Table 2: Waypoints generated by the plume tracking algorithm for the centroid tracking vehicle for both days.

\begin{tabular}{|ccc|ccc|}
\hline Day 1 & Latitude (N) & Longitude (E) & Day 2 & Latitude $(\mathrm{N})$ & Longitude $(\mathrm{E})$ \\
\hline 1 & 33.5350 & -118.5600 & 1 & 33.5350 & -118.5600 \\
2 & 33.5612 & -118.5526 & 2 & 33.5081 & -118.5584 \\
3 & 33.5759 & -118.5254 & 3 & 33.5332 & -118.5466 \\
4 & 33.5530 & -118.5424 & 4 & 33.5201 & -118.5183 \\
5 & 33.5693 & -118.5167 & 5 & 33.4974 & -118.5357 \\
\hline
\end{tabular}

Table 3: Waypoints generated by the plume tracking algorithm for the boundary tracking vehicle for both days.

Upon completion of the day one mission, the gliders resumed their previous missions of gathering communication data while we awaited the following day's ROMS prediction. The entire process mentioned above was repeated beginning at approximately $2200 \mathrm{Z}$ on May 12, 2009. The initial plume delineation, glider surfacing locations and projected trajectories are given in Figs. 12(a) - 12(e). The computed waypoints for the centroid and boundary tracking vehicles are given in the right-hand columns of Tables 2 and 3, respectively. Day 2 of the mission completed shortly after 1400Z on May 13, 2009. Note that at the start of day 2, we re-delineated the plume differently from the final predicted configuration of day 1. This is applicable in practice because we cannot assume ROMS to be $100 \%$ accurate, and we have a time gap between the start of the sampling missions where we expect the plume to further evolve. Additionally, we are forecasting the evolution of a plume based on the predicted paths of multiple, unconnected, Lagrangian drifters, which may not behave exactly like a contiguous ocean feature.

Overall, this two-day mission validated both the multi-vehicle applicability as well as the ability to close the loop on the technology toolchain from ROMS to path planning to field AUV and back to ROMS. This further motivates our efforts to prepare for a large-scale deployment with hopes of locating and tracking a real plume or HAB.

\section{Simulation}

Multiple environmental agencies, local and regional policy makers, universities and outreach groups in Southern California collaborate together to assess the status of streams, estuaries, beaches, and marine environments in Southern California. More than 90 local organizations contribute to this biannual effort called the Southern California Bight Regional Marine Monitoring Program, which includes assessment in areas of Coastal Ecology, Water Quality, Rocky Subtidal, Areas of Special Biological Significance, and Shoreline Microbiology. Conducting large-scale, collaborative, regional assessments is a benefit to all agencies due to the widespread appeal and shared data products between all contributors. Rather than making comparisons to a small number of control sites, agencies are able to compare local results to the entire breadth of natural variability inherent to the ecosystem. This allows regulators to target resources where action is most needed. A few main questions posed for the study are the percent of the Southern California mainland shelf area exhibiting signs of human disturbance, or the number of stream-miles impacted by anthropogenic activities, or the long term effects of rapid urbanization in Southern California related to the frequency of occurrence of HAB events. 


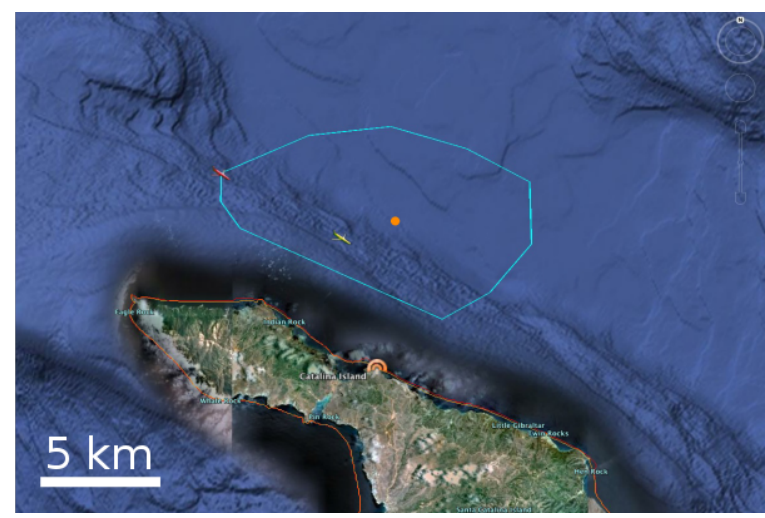

(a) $T=0 \mathrm{hrs}$

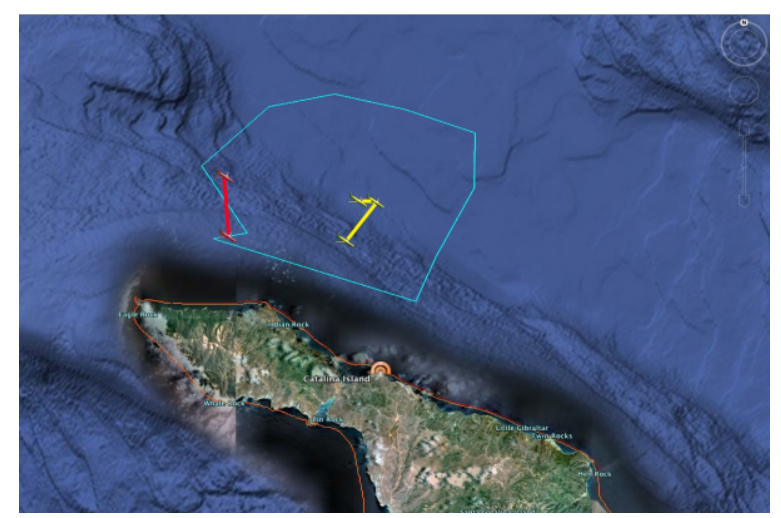

(b) $T=4 \mathrm{hrs}$

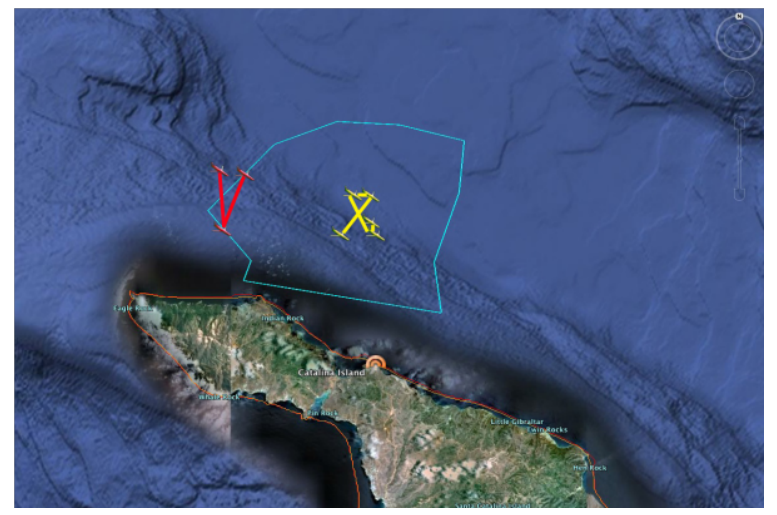

(c) $T=8 \mathrm{hrs}$

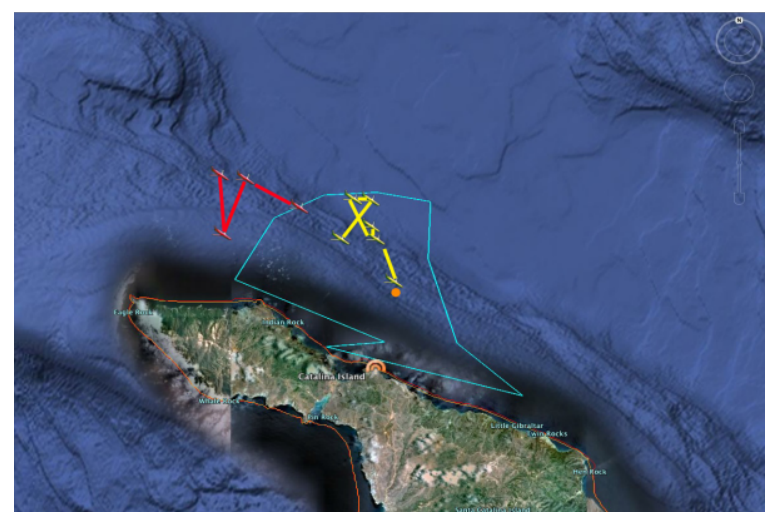

(d) $T=12 \mathrm{hrs}$

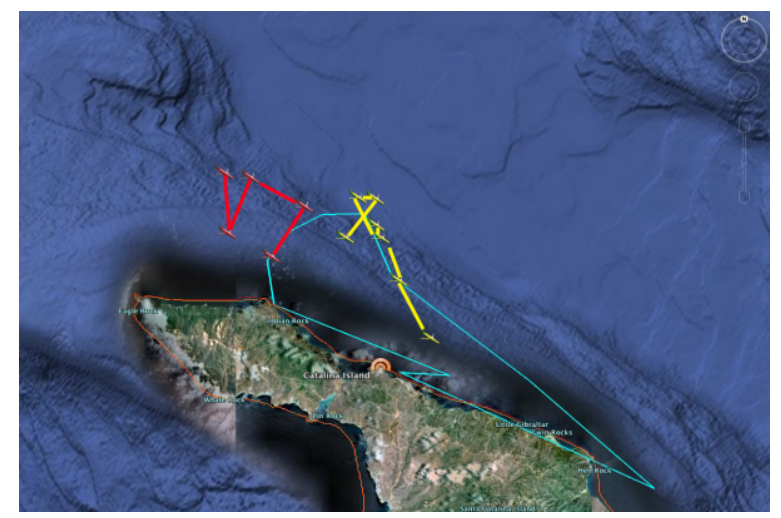

(e) $T=16 \mathrm{hrs}$

Figure 12: Centroid and boundary tracking experiment, Day 2. The scale for all images is given in Fig. 12(a). Images created by use of Google Earth. 
The next Southern California Bight Regional Marine Monitoring Program, commonly referred to as Bight 2010, is scheduled to take place from late-January through early-April 2010. The CINAPS group at USC will be a contributor in the Bight ' 10 survey. One planned aspect of our contribution will be continuous operation of four ASGs in the SCB for the entire three-month program. During this time, we are planning to implement the techniques presented in this work to retask currently operating ASGs in the field to track and monitor a freshwater plume, waste water outfall or HAB event. Through careful planning and a bit of luck, we hope to capture the conditions in the SCB leading up to and development of a $\mathrm{HAB}$ event. To this end, we present a simulation experiment with four ASGs tracking a feature of interest off the coat of Newport Beach, CA.

In this scenario, we offset the start times of the four vehicles to emulate asynchronous surfacing and communication with the vehicles or to simulate deploying the vehicles at multiple locations for the specific feature of interest. At $T=0$, we deploy two vehicles, one centroid tracker and one boundary tracker, at the southern extent of the plume. At $T=2$ hours we deploy a boundary tracking vehicle on the predicted western boundary of the plume. Finally at $T=4$ hours, we start a boundary tracking vehicle at the predicted northern boundary of the plume. The initial delineation and location of the first two gliders is presented in Fig. 13(a). The evolution of the plume with vehicle trajectories is presented in Fig. 13(a) - Fig. 14(f). The trajectories of the vehicles are the expected trajectories of the ASGs, projected to the ocean surface. Note that in Figs. 13(a) - 14(f), we only display a trajectory for a vehicle at the hour when it surfaces due to the offset in start times. The centroid of the predicted plume is depicted by the orange dot, the centroid tracking vehicle is given by the yellow icons and trajectory lines and the boundary tracking vehicles are denoted by the red icons and trajectory lines. The predicted extent of the plume is delineated by the white polygon.

The trajectory design is based upon model predictions, and we are familiar with the deployment area, we do have an a priori understanding of the general direction the feature should travel. This knowledge can be used to select the initial locations of the vehicles based upon the information to be gathered and areas of interest within the feature. In this example, since we see a rather fast moving feature in the southeast direction, we choose to start the centroid tracker on the southern extent of the feature. Thus, we do not try and chase the area we are interested in sampling, and have a higher probability of collecting data of high scientific merit. Based on the movement of the feature, the centroid tracking vehicle (yellow) actually completes a U-shape trajectory, and from $T=12-16$ hours cannot keep up with the feature, based on our constant speed assumption. We also see the speed of the feature since the boundary tracking vehicles (red) traverse more of a straight line path than the zig-zag seen with a slower-moving feature as in Figs. 11(f) and 12(e). Overall, the trajectories presented here, and in the previous deployment sections, do not resemble those that a human operator would design. However, the trajectories do guide the vehicles through a large portion of the plume during its predicted evolution, thus increasing the probability of collecting high-valued data for both the marine biology community and the modeling community alike.

\section{Conclusions and Future Work}

In the study of path planning for field robots, designing the trajectory is usually less than half the battle, the real challenge comes in the implementation. This is exaggerated when dealing with underwater robots due to the complex environment. Poorly understood ocean dynamics, difficulty in localization and extreme conditions all contribute to the struggle of successful implementation of a planned mission for an AUV.

Generating effective sampling strategies to study ocean phenomena is a challenging task that can be approached from many different angles. Here, we presented a method to exploit multiple facets of technology to achieve our goal. Utilizing an ocean model, an embedded sensor network and an AUV, we were able to construct a technology chain which designs a trajectory to track areas of scientific interest within a chosen oceanic feature for a chosen period of time. This toolchain was used to design a single-vehicle mission to track a plume's centroid, and generate the trajectories for a multi-vehicle mission for simultaneous sampling at the centroid as well as along the boundary of a given feature. We presented iterated field trials to demonstrate closing the loop of the technology chain developed as well as to give a proof-of-concept of the method presented. This paper has demonstrated that we have implemented the collaboration and technology chain required to perform complex field experiments to track dynamically evolving ocean features. Research is now active to improve upon the presented 


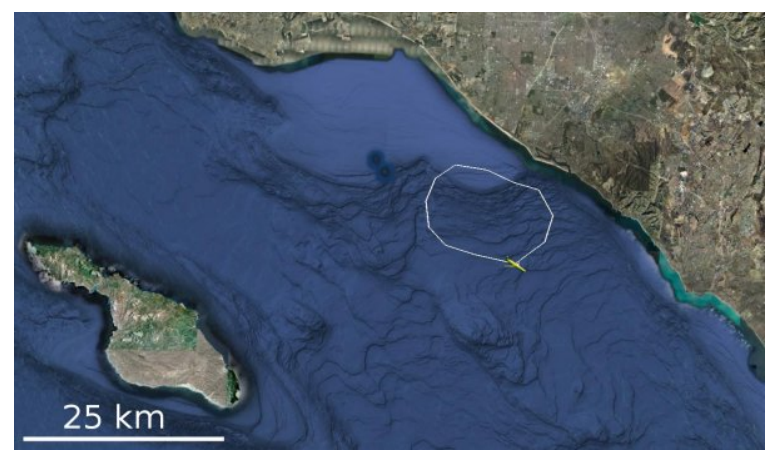

(a) $T=0 \mathrm{hrs}$

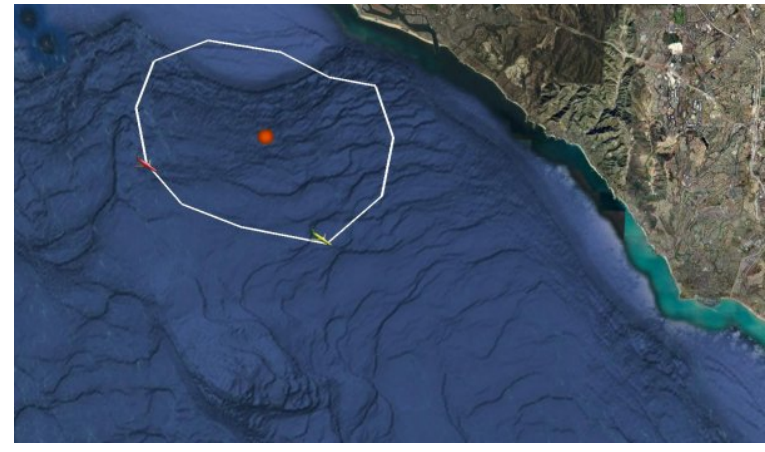

(c) $T=2 \mathrm{hrs}$

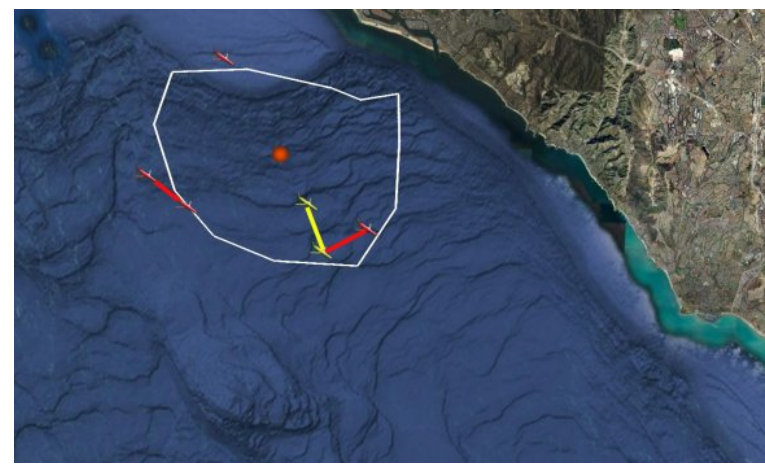

(e) $T=6 \mathrm{hrs}$

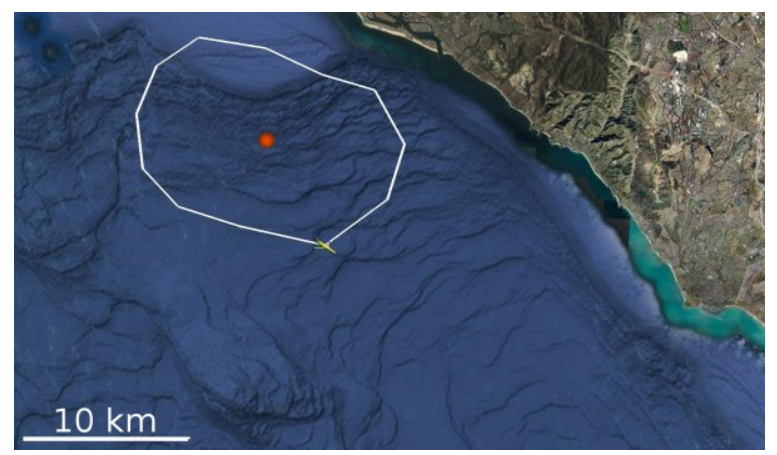

(b) $T=0 \mathrm{hrs}$

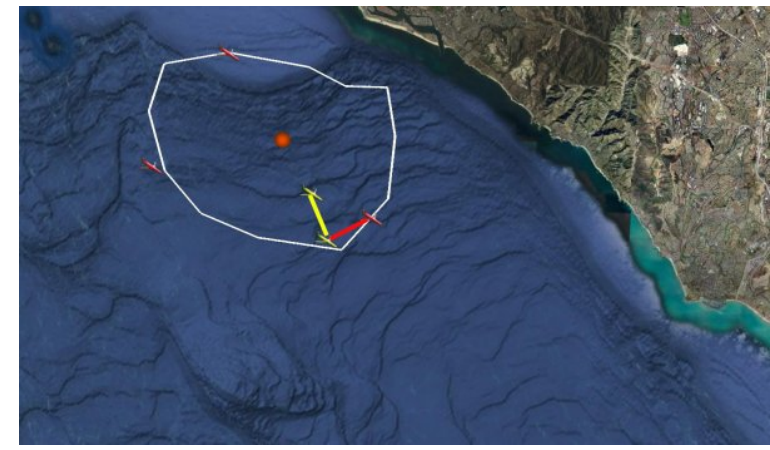

(d) $T=4 \mathrm{hrs}$

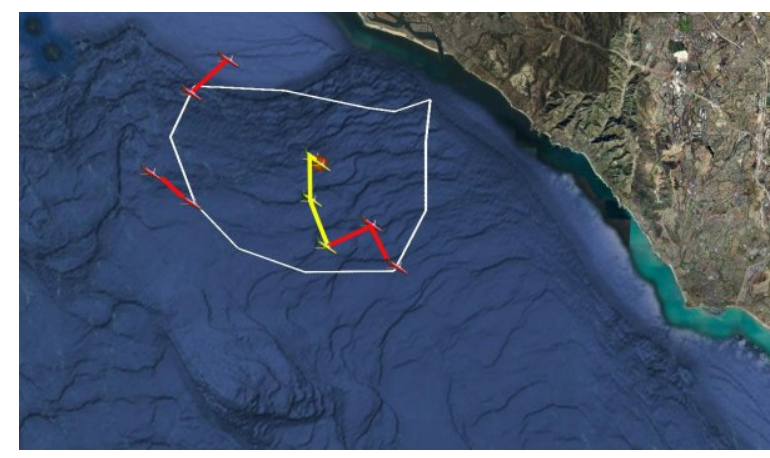

(f) $T=8 \mathrm{hrs}$

Figure 13: Centroid and boundary tracking simulation experiment from initialization to $T=8$ hours. The scale given in Fig. 13(b) is the same for Figs. 13(c) - 13(f). Images created by use of Google Earth. 


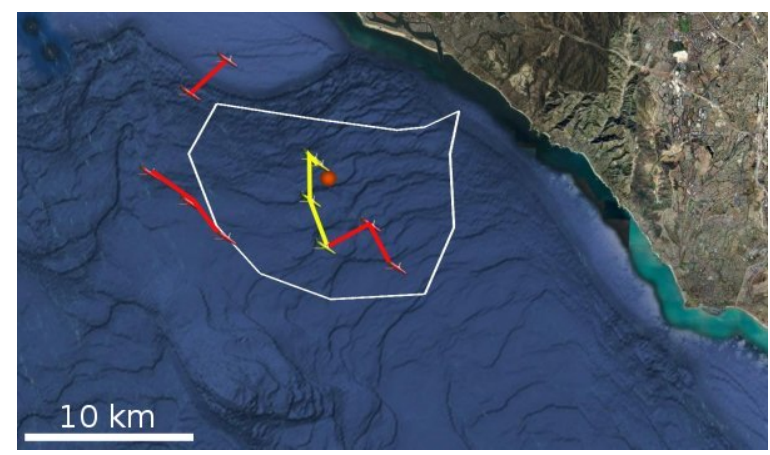

(a) $T=10 \mathrm{hrs}$

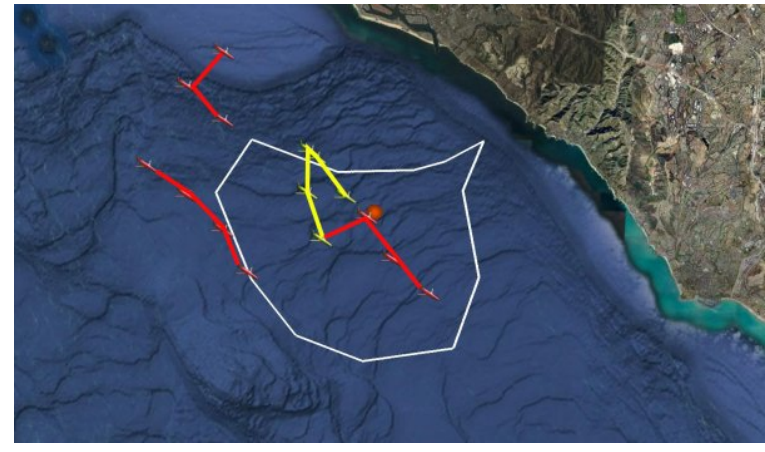

(c) $T=14 \mathrm{hrs}$

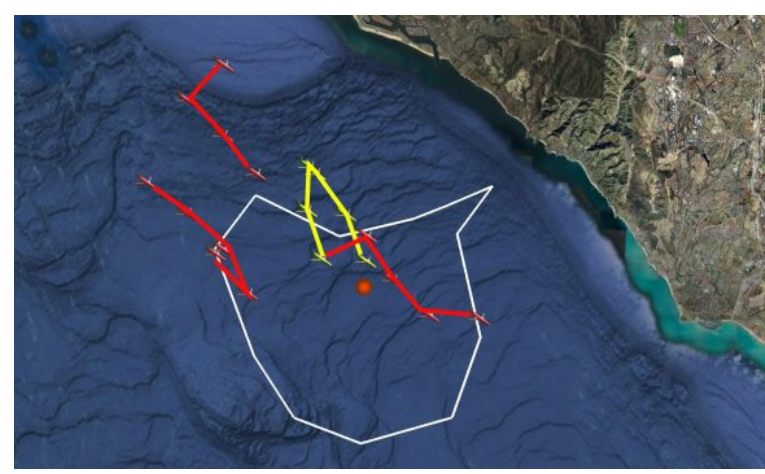

(e) $T=18 \mathrm{hrs}$

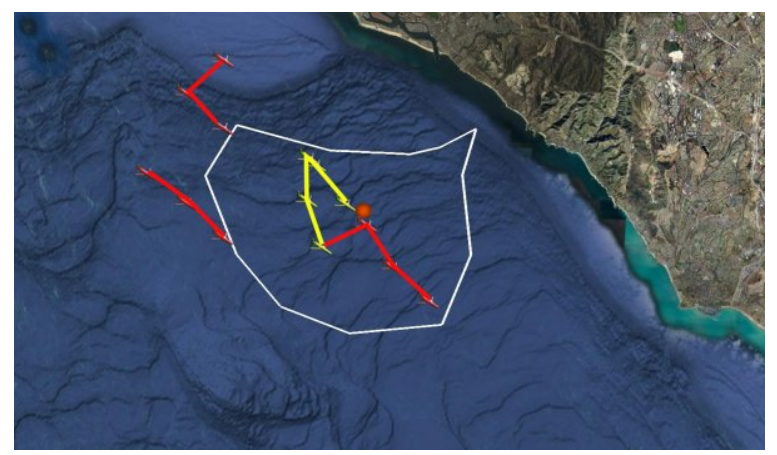

(b) $T=12 \mathrm{hrs}$

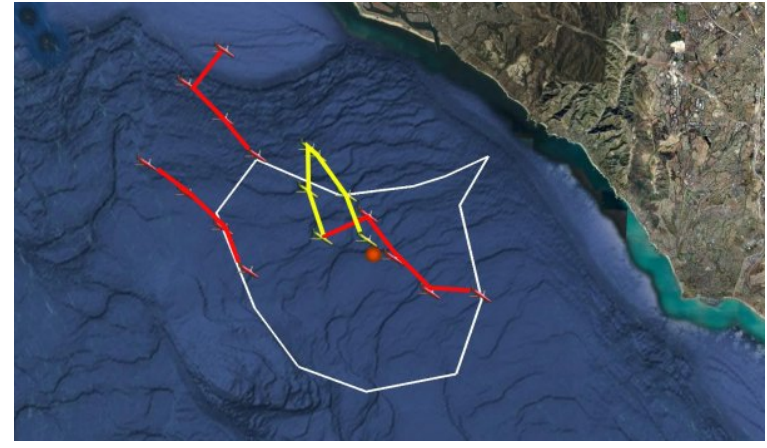

(d) $T=16 \mathrm{hrs}$

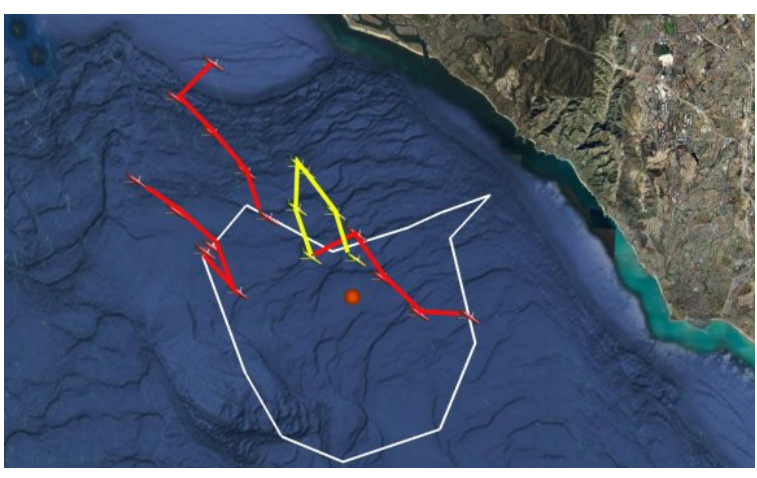

(f) $T=20 \mathrm{hrs}$

Figure 14: Centroid and boundary tracking simulation experiment from $T=10$ hours to completion. The scale for all images is given in Fig. 14(a). Images created by use of Google Earth. 
waypoint generation algorithms to incorporate ROMS 4-D velocity predictions to improve the accuracy of the implementation (Smith et al. 2010). Additionally, with this research, we are interested in assessing the accuracy of ROMS velocity predictions in a coastal environment.

The main implementation issue is the ability of the ASG to accurately navigate to a given waypoint. In general, this is a widely-studied problem in underwater robotics. Here, this artifact is a result of the waypoint selection algorithm only solving the 2-D problem, and ignoring the dynamics of the glider and the complex ocean environment. Research is active to extend these results to incorporate the kinematic and dynamic models of the glider and extend this from a planar to a 3-D path planning algorithm and trajectory generation toolchain. A long term goal of this effort is to utilize the embedded sensor network presented in (Smith et al. 2009c) and (Pereira et al. 2009) to enable real-time, optimal trajectory design, based on ocean model predictions, to gather in situ measurements of interesting and evolving ocean features and phenomena while additionally increasing the skill of regional ocean models. As previously mentioned, this will come with deployment of actual drifters simulating a propagating feature, as well as during Bight 2010, for which we are prepared to track an actual event.

As discussed in Sect. 7, a more immediate goal is our participation in the Southern California Bight Regional Marine Monitoring Program (Bight 2010). Currently, we are experiencing weak El Niño conditions, as equatorial Pacific, sea-surface temperatures remained above average through August 2009. These conditions are expected to strengthen through winter 2009-2010 in the Northern Hemisphere (Climate Prediction Center 2009), and will impact Southern California with more frequent and intense storm events. From the discussion in Sects. 2.1 and 2.2, we can infer that the potential for the formation of conditions that may promote an algal bloom is increased over the next year. Such information presents a greater opportunity to retest and validate our methods and algorithms in a real scenario. Thus, we are motivated to further develop our algorithms to steer AUVs into locations of high scientific merit with regard to $\mathrm{HAB}$ research and help ocean scientists better understand these complex phenomena, while additionally assessing the prediction capabilities of regional ocean models.

\section{Acknowledgements}

This work was supported in part by the NOAA MERHAB program under grant NA05NOS4781228 and by NSF as part of the Center for Embedded Network Sensing (CENS) under grant CCR-0120778, by NSF grants CNS-0520305 and CNS-0540420, by the ONR MURI program (grants N00014-09-1-1031 and N00014-08-1-0693) by the ONR SoA program and a gift from the Okawa Foundation. The ROMS ocean modeling research described in this publication was carried out by the Jet Propulsion Laboratory (JPL), California Institute of Technology, under a contract with the National Aeronautics and Space Administration (NASA).

The authors acknowledge Carl Oberg for his work with glider hardware making field implementations possible and simple. We thank Ivona Cetinic Arvind Pereira and Hörður Heiðarsson for their efforts in the glider deployment and communications during the missions.

\section{References}

Anderson, D. (2008), 'Harmful algae', http://www.whoi.edu/redtide/page.do?pid=9257. viewed February 2009.

Cetinic, I., Jones, B., Moline, M. \& Schofield, O. (2008), 'Resolving urban plumes using autonomous gliders in the coastal ocean', Journal of Geophysical Research/Americal Geophysical Union .

Chao, Y., Li, Z., Farrara, J., McWilliams, J. C., Bellingham, J., Capet, X., Chavez, F., Choi, J.-K., Davis, R., Doyle, J., Frantaoni, D., Li, P., Marchesiello, P., Moline, M., Paduan, J. \& Ramp, S. (2008), 'Development, implementation and evaluation of a data-assimilative ocean forecasting system off the central california coast', Deep-Sea Research II .

Climate Prediction Center (2009), 'El Niño/Southern Oscillation (ENSO) diagnostic discussion', http: //www.cpc.noaa.gov/products/analysis_monitoring/enso_advisory/ensodisc.html. viewed September 2009.

Dailey, M. D., Reish, D. J. \& Anderson, J. W., eds (1993), Ecology of the Southern California Bight, University of California Press, Berkley. 
Davis, R. E., Ohman, M., Rudnick, D., Sherman, J. \& Hodges, B. (2008), 'Glider surveillance of physics and biology in the southern california current system', Limnol. Oceanogr. .

Fiorelli, E., Leonard, N., Bhatta, P., Paley, D., Bachmayer, R. \& Fratantoni, D. (2006), 'Multi-auv control and adaptive sampling in monterey bay', IEEE Journal of Oceanic Engineering 31(4), 935948.

Graver, J. (2005), Underwater Gliders: Dynamics, Control and Design, PhD thesis, Princeton University.

Gray, A. M., Lee, C., D’Asaro, E. A., Perry, M. J., Fennel, K., Briggs, N., Rehm, E., Sackmann, B. S., Sieracki, M. E., Poulton, N. \& Gudmundsson, K. (2008), The 2008 north atlantic spring bloom experiment: Observations of a stationary eddy on the eastern flank of the reykjanes ridge, in 'American Geophysical Union, Fall Meeting 2008'. Abstract \# OS31A-1252.

Jet Propulsion Laboratory (2009), 'JPL THREDDS Data Server catalog', http://ourocean.jpl.nasa . gov:8080/thredds/catalog.html. viewed September 2009.

Kennedy, V. S., Twilley, R. R., Kleypas, J. A., J. H. Cowan, J. \& Hare, S. R. (2002), Coastal and marine ecosystems \& global climate change, in 'Pew Center on Global Climate Change', p. 52.

Kudela, R. M., Ryanb, J. P., Blakelya, M. D., Lanea, J. Q. \& Petersona, T. D. (2008), 'Linking the physiology and ecology of cochlodinium to better understand harmful algal bloom events: A comparative approach', Harmful Algae/Elsevier 7(3), 278-292.

Leonard, N. E. \& Graver, J. G. (2001), 'Model-based feedback control of autonomous underwater gliders', IEEE Journal of Oceanic Engineering, Special Issue on Autonomous Ocean-Sampling Networks 26(4), 633-645.

Leonard, N. E., Paley, D., Lekien, F., Sepulchre, R., Fratantoni, D. M. \& Davis, R. (2007), Collective motion, sensor networks and ocean sampling, in 'Proceedings of the IEEE', Vol. 95(1), pp. 48-74.

Li, Z., Chao, Y., McWilliams, J. \& Ide, K. (2008a), 'A three-dimensional variational data assimilation scheme for the regional ocean modeling system', Journal of Atmospheric and Oceanic Technology 25, 2074-2090.

Li, Z., Chao, Y., McWilliams, J. \& Ide, K. (2008b), 'A three-dimensional variational data assimilation scheme for the regional ocean modeling system: Implementation and basic experiments', Journal of Geophysical Research 113(C05002).

Paley, D., Zhang, F. \& Leonard, N. E. (2007), 'Control of coordinated patterns for ocean sampling', International Journal of Control, special issue on Navigation, Guidance and Control of Uninhabited Underwater Vehicles 80(7), 1186-1199.

Paley, D., Zhang, F. \& Leonard, N. E. (2008), 'Cooperative control for ocean sampling: The glider coordinated control system', IEEE Transactions on Control Systems Technology 16(4), 735-744.

Pereira, A., Heidarsson, H., Caron, D., Jones, B. \& Sukhatme, G. (2009), An implementation of a communication framework for the cost-effective operation of slocum gliders in coastal regions, in 'Proceedings of The $7^{\text {th }}$ International Conference on Field and Service Robotics', Cambridge, MA.

Schnetzer, A., Miller, P. E., Schaffner, R. A., Stauffer, B., Jones, B. H., Weisberg, S. B., DiGiacomo, P. M., Berelson, W. M. \& Caron, D. A. (2007), 'Blooms of pseudo-nitzschia and domoic acid in the san pedro channela and los angeles harbor areas of the southern california bight, 2003-2004', Harmful Algae 6(3), 372-387.

Schofield, O., Kohut, J., Aragon, D., Creed, E., Graver, J., Haldman, C., Kerfoot, J., Roarty, H., Jones, C., Webb, D. \& Glenn, S. (2007), 'Slocum gliders: Robust and ready', Journal of Field Robotics 24(6), 473-485.

Shchepetkin, A. \& McWilliams, J. (1998), 'Quasi-monotone advection schemes based on explicit locally adaptive dissipation', Monthly Weather Review 126, 1541-1580.

Shchepetkin, A. \& McWilliams, J. (2005), 'The regional oceanic modeling system (ROMS): a splitexplicit, free-surface, topography-following-coordinate oceanic model', Ocean Modeling 9, 347-404. doi:10.1016/j.ocemod.2004.08.002.

Smith, R. N., Chao, Y., Jones, B. H., Caron, D. A., Li, P. P. \& Sukhatme, G. S. (2009a), Trajectory design for autonomous underwater vehicles based on ocean model predictions for feature tracking, in 'Proceedings of The $7^{\text {th }}$ International Conference on Field and Service Robotics', Cambridge, MA. 
Smith, R. N., Das, J., Heidarsson, H., Pereira, A., Caron, D. A., Jones, B. H. \& Sukhatme, G. S. $(2009 b)$, Implementation of an embedded sensor network for the coordination of slocum gliders for coastal monitoring and observation, in 'Workshop on UnderWater Networks; ACM SenSys 2009', Berkeley, CA. accepted, to appear.

Smith, R. N., Das, J., Heidarsson, H., Pereira, A., Cetinić, I., Darjany, L., Ève Garneau, M., Howard, M. D., Oberg, C., Ragan, M., Schnetzer, A., Seubert, E., Smith, E. C., Stauffer, B. A., ToroFarmer, G., Caron, D. A., Jones, B. H. \& Sukhatme, G. S. (2009c), 'The Center for Integrated Networked Aquatic PlatformS (CINAPS): Observing and monitoring the southern california bight', IEEE Robotics and Automation Magazine, Special Issue on Marine Robotics Systems . Submitted.

Smith, R. N., Pereira, A. A., Chao, Y., Li, P. P., Caron, D. A., Jones, B. H. \& Sukhatme, G. S. (2010), Autonomous underwater vehicle trajectory design coupled with predictive ocean models: A case study, in 'IEEE International Conference on Robotics and Automation', Anchorage, Alaska. Submitted.

U.S. Commission on Ocean Policy (2004), An Ocean Blueprint for the 21st Century, Washington, D.C. ISBN\#0-9759462-0-X, http://oceancommission.gov/.

Vu, Q. (2008), 'JPL OurOcean portal', http://ourocean.jpl.nasa.gov/. viewed February 2009.

Warrick, J. A. \& Fong, D. A. (2004), 'Dispersal scaling from the world's rivers', Geophysical Research Letters 31(4), L04301. DOI: 10.1029/2003GL019114.

Webb Research Corporation (2008), http://www. webbresearch.com/slocum.htm. viewed May 2008.

Wood, E., Schnetzer, A., Benitez-Nelson, C., Anderson, C., Berelson, W., Burns, J., Caron, D., Ferry, J., Fitzpatrick, E., Jones, B., Miller, P., Morton, S. \& Thunell, R. (2009), 'The toxin express: Rapid downward transport of the neurotoxin domoic acid in coastal waters', Nature Geosciences . 